\title{
On the gravitational suppression of hydrothermal modes in liquid layers with a blockage on the bottom wall
}

\author{
Marcello Lappa \\ Department of Mechanical and Aerospace Engineering, University of Strathclyde \\ James Weir Building, 75 Montrose Street, Glasgow, G1 1XJ, UK \\ email: marcello.lappa@strath.ac.uk
}

\begin{abstract}
The present analysis follows and integrates author's previous work (Lappa M., 2017, Int. J. Thermal Science, 118: 303-319), where the main focus was on buoyancy flow of semiconductor and superconductor melts in geometries with an obstruction located on the bottom. Here we consider hybrid thermogravitational/thermocapillary convection in the case of high-Pr materials for which the typical disturbances are represented by the so-called hydrothermal waves. By means of two-dimensional and three-dimensional numerical simulations, we show that the presence of a blockage in the physical domain can significantly change the dynamics of these waves in some regions of the space of parameters. More precisely, in some specific conditions their velocity of propagation can be modulated and even reduced to zero causing replacement of rolls travelling in the system with disconnected segments of stationary waves that originate from the stations where sudden variations of the geometry occur.
\end{abstract}

Keywords: Hydrothermal waves, Marangoni convection, buoyancy convection

\section{Introduction}

There is a long tradition of studies in the literature exploring the intricacies underpinning the socalled mixed thermocapillary-thermogravitational flow stability problem (see, e.g., Schwabe and Metzger [1]; Saghir and Maffei [2]; Melnikov et al., [3,4]; Peng, Li, Shi and Imaishi [5]; Shevtsova et al. [6,7], Lappa [8,9] and references in all these papers). Many of these analyses have addressed the case of a layer of infinite extent with top free surface subjected to a horizontal temperature gradient. Other valuable studies have concentrated on laterally heated finite-size cavities for which boundaries do play a non-negligible role on the resulting dynamics.

We have learned that, between the pure gravitational (buoyant) and Marangoni (thermocapillary) flows a variety of intermediate situations are possible. In particular, one of the most remarkable achievements obtained in recent years is the realization that these dynamics and the related transitional stages of evolution are largely determined by a sort of very complex interplay between the characteristic modes of instabilities pertaining to these two limiting classes of flow. In some cases these modes are inextricably intertwined; in other circumstances the hybrid flow seems to retain some of the fundamental properties of either parent categories of flow.

Relevant examples along these lines are the linear stability analyses by Gershuni et al. [10] and Parmentier et al. [11], who concentrated on configurations with thermally conducting or adiabatic horizontal boundaries, respectively; while for a third companion case considering a free surface 
affected by a given heat exchange rate (quantified via the so-called Biot number), the reader may consider the subsequent work by Mercier and Normand [12].

Given the high sensitivity that hybrid convection has proven to exhibit to the type of thermal conditions defined by the three variants mentioned above, to the Prandtl number and the relative importance of buoyancy and surface-tension-driven effects (measured by the nondimensional parameter $\mathrm{W}=\mathrm{Ma} / \mathrm{Ra}$ where $\mathrm{Ma}$ and $\mathrm{Ra}$ are the typical Marangoni and Rayleigh numbers), this subject still hampers physicists' and engineers' quest to map precisely the response of these systems in the space of parameters. The complexity of the problem is further reinforced by other influential factors, such as the effective shape of the container hosting the fluid and the kinematic conditions established at the bottom wall. Towards the end to assess the ability of these flows to break specific spatial "symmetries", most of existing studies have been based on the paradigm of a series of physical domain with well-defined, regular and 'classical” shapes (and a recognized major concern with the practical applicability of this approach is the non-straightforward way by which such knowledge should be translated towards practical applications).

For the simplest case of plane-parallel flow (which define a class of basic solutions for the present problem as the parameter $W$ is varied in the range $0 \leq \mathrm{W} \leq \infty)$, some fundamental information can be found, e.g., in the recent review of available analytic solutions by Lappa [13]. As illustrated therein, for $0 \leq \mathrm{W} \leq 0.25$ the typical velocity profile of the flow established in an infinite layer has an inflection point whose position depends on $\mathrm{W}$ (this point moves toward the upper free boundary when $\mathrm{W}$ increases from zero and it disappears when $\mathrm{W}>0.25$ ). Moreover, for the specific case of conducting boundaries the temperature profiles display two zones of unstable stratification near the boundaries and one zone of stable stratification in the middle of the layer having location and thickness depending on $\mathrm{W}$.

Using as basic flow analytic solutions of such a kind for the case of horizontal conducting boundaries, Gershuni et al. [10] found the mechanisms leading to unstable hybrid convection for $\mathrm{W}<\mathrm{O}\left(10^{-1}\right)$ to be essentially the same as in the pure buoyancy case ( $\mathrm{W}=0$ ) (the allowed modes of convection being oscillatory 2D hydrodynamic modes, OLR waves and Rayleigh modes).

Interestingly, even for $\mathrm{W}>1$, these authors did not report waves with features of the hydrothermal type (HTW), like those that should be expected for pure Marangoni flow (as originally predicted by earlier linear stability analyses). As extensively discussed, e.g., by Smith and Davis [14], the basic mechanism and feedback loops at the root of the HTW phenomena are intimately related to the coupling of surface stresses and surface temperature disturbances; as a natural consequence, they are suppressed if either $\mathrm{W}=0$ (no Marangoni effects) or the free surface is conducting (in such a case, in fact, no temperature disturbances are possible at the free interface because the temperature distribution is fixed). By contrast, waves of such a kind were clearly identified in the subsequent linear stability analysis by Parmentier et al. [11] for hybrid buoyancy-Marangoni convection in layers with adiabatic horizontal boundaries and a wide range of variation of the Prandtl number $\mathrm{O}\left(10^{-2}\right) \leq \mathrm{Pr} \leq \mathrm{O}(10)$. 
Apart from such theoretical studies, over the years several experimental analyses have been produced essentially for transparent liquids and liquid layers with rectangular section. Often counter-intuitive results have been yielded. Though Riley and Neitzel [15] could clearly observe HTW phenomena in a rectangular pool $L_{x}=5.3[\mathrm{~cm}], L_{z}=5.5[\mathrm{~cm}]$ for silicone oil $(\operatorname{Pr}=14)$ for sufficiently thin layers $(\mathrm{d}<1.25[\mathrm{~mm}])$, states not corresponding to the propagation of waves were reported by many other authors Villers and Platten [16], De Saedeleer et al. [17] as a result of a back transition from oscillatory to steady states produced by an increase in the thickness of the layer.

Due to such studies, eventually, it has been recognized that a challenge in pushing the boundaries of our current understanding of these phenomena relates to accurately capturing the emerging behavior in relation to the relative importance of surface-tension driven and buoyancy effects and the specific design of the container hosting the fluid.

Most of the available results for transparent liquids (Villers and Platten [16] for Pr=4.2; Ezersky et al. [18] for $\operatorname{Pr}=60$; Daviaud and Vince [19] for $\operatorname{Pr}=10.3$; Gillon and Homsy [20] for Pr=9.5; De Saedeleer et al. [17] for $\operatorname{Pr}=15$; Braunsfurth and Homsy [21] for $\operatorname{Pr}=4.4$; Garcimartìn et al. [22] for $\operatorname{Pr}=10,15$ and 30; Pelacho and Burguete [23] and Pelacho et al. [24] for Pr=10.3; etc.) have been summarized (together with the related experimental setups, i.e. length, width and height of the containers used) in the analysis by Burguete et al. [25]. In such a context, it is also worth recalling that Pelacho et al. [26] performed a series of experiments specifically designed to characterize the emergence of waves in rectangular containers whose dimensions could be continuously changed.

Still using the present opportunity as a means to present a brief account of the historical perspective relating to these subjects, it should also be mentioned that additional insights were provided by Ospennikov and Schwabe [27], given their ability to demonstrate that HTWs can artificially be damped (leading to stationary rolls) in experimental configurations where the return flow does not exist (in practice, in their experiments, the return flow could be suppressed using channels and side channels with lower flow resistance compared to that of the return flow).

More recently, other authors re-examined the problem in the framework of relevant linear or nonlinear analysis techniques. As an example, using linear stability analysis, Chan and Chen [28] concentrated on the conditions relating to the experiments by Riley and Neitzel [15]. In qualitative agreement with the earlier findings by Parmentier et al. [11] for $\operatorname{Pr}=7$, they found that for $\operatorname{Pr}=13.9$ an increase in the relative importance of buoyancy can cause a decrease in the inclination angle $\Phi$ of the HTWs with respect to the basic flow. Most interestingly, they could even identify two different threshold values of the Rayleigh number such that, beyond the first threshold oblique waves are taken over by transverse $\left(\Phi=0^{\circ}\right)$ waves propagating in the upstream direction while, when the second threshold is exceeded, these transverse waves may even reverse their sense of propagation. Other relevant and recent efforts are those by Sáenz et al. [29]. To the best of our knowledge, these are the only existing simulations where the problem was addressed directly in terms of solution of the multiphase non-linear equations accounting for both the liquid phase and the overlying layer of gas. More precisely, these authors used a moving-boundary technique relying 
on the VOF (Volume of Fraction) strategy. In this way, they could observe that the outcomes of the instability of mixed Marangoni-buoyancy convection are not limited to the HTWs as these instabilities can also induce interface deformation, i.e. physical waves propagating in the system with the same angle of propagation but with a phase-lag with respect to the original HTW signal. This specific line of inquiry was further expanded by Sáenz et al. [30] by taking into account the evaporation of the liquid into the gas phase and related latent heat effects. As revealed by this study, the HTW-induced velocity field in the gas can deeply affect the local evaporation flux and the vapor distribution above the interface. Interestingly, due to a phase shift in the convective phenomena occurring in the liquid and in the gas, the concentrations of vapor tend to be higher above the regions where the temperature induced locally by the HTW is relatively low.

Very recently, Kowal et al. [31] have filled another existing gap, that is, the lack of information about the influence of the thermal and kinematic conditions established at the other end of the liquid domain, i.e. the bottom wall. While all of previous studies had considered classical no-slip (no velocity) conditions, these authors implemented an alternate model relying on a mixed boundary condition (of the Robin type) at the bottom (formally introduced by setting the derivative of horizontal velocity in direction perpendicular to the wall equal to the local velocity value times a “slip” parameter $\varphi$, ranging beween 0 (free slip) and $\infty$ (no slip)). According to this study (yet a linear stability analysis), partial slip generally lowers the critical values of the Marangoni number and makes waves travelling in the direction perpendicular to the basic flow less likely to occur. Moreover, two-dimensional (transverse) hydrothermal waves become the preferred mode of instability for intermediate values of the Prandtl numbers and low Biot numbers (the range of Prandtl numbers for which these waves can exist shrinking with the slip parameter).

Leaving aside for a while the details of the specific configurations or strategies of attack implemented by different research groups, all these efforts (providing disconnected qualitative and quantitative glimpses of wide-ranging behaviors) could be summarized as follows: the hydrothermal waves typical of Marangoni flow can be weakened or damped if one or more of the following conditions are satisfied, namely: 1) the free surface of the liquid behaves as a purely thermally conducting boundary (i.e. a fixed linear horizontal temperature profile is established there); 2) the geometry of the container geometry satisfies certain requirements; 3) though the parameter $\mathrm{W}$ is smaller than 1 , buoyancy effects are sufficiently intense with respect to surfacetension driven ones. The present work has been specifically conceived along these lines. More specifically, we aim to show that the combined "use" of buoyancy (decreasing values of W) and specific asymmetries in the shape of the shallow container hosting the liquid can be used to induce circumstances for which such waves are mitigated (in terms of amplitude) or completely suppressed (leading to a recovery of a steady state).

In particular, we concentrate on containers with varying crossing section, which have recently attracted interest as new archetypal systems for the improvement of our fundamental understanding of both thermogravitational and thermocapillary flows. The present study has its root in an already running line of inquiry which includes, e.g., refs [32-34]. 
As HTWs are allowed to form for both cases with adiabatic and thermally conducting conditions on the bottom wall, here we address both circumstances. More precisely, this paper follows and integrates the results presented by Lappa [35] on the effect of sudden changes in the cross-section of the liquid container on the emergence of the HTW in the case of pure microgravity conditions (no buoyancy).

A parametric study is conducted for mixed thermogravitational-thermocapillary convection. In particular, the potential of the proposed framework is demonstrated first by conducting (2D) simulations given the significant advantages provided by such an approach in terms of computational cost, and then by fully three-dimensional (3D) simulations used to confirm the main outcomes of the 2D study (by probing the system response in selected representative regions of the space of parameters). In this regard the present parametric investigation may be also regarded as a fundamental investigation complementing that by Ref [36]. In that work the target was the companion problem represented by the mitigation of oscillatory hydrodynamic modes of gravitational (buoyancy) nature in containers with a topography on the bottom wall due to the presence of surface-tension driven effects (a kind of "mirror" subject with respect to that being addressed here). A kinship also exists with regard to another important category of problems, that is, the stability of thin films flowing down an inclined wall in the presence of Marangoni effects. Indeed, this topic has been investigated over recent years taking into account the presence of a topography and for different thermal boundary conditions at the wall. It has been found that stabilizing or destabilizing effects can be produced by the complex interplay of gravitational, capillary, geometrical and thermal effects [37-40].

\section{Mathematical Model and Numerical Method}

\subsection{The System}

In line with the two earlier studies [35,36], we focus on a shallow rectangular cavity with free liquid-gas interface along the $\mathrm{x}$ axis bounded laterally by two solid walls at different temperatures (one cooled, the other heated) having height $\mathrm{d}$.

As anticipated, this canonical geometry (the so-called R-F system, Laure and Roux [41], see Fig. 1) can be made more interesting in terms of expected fluid-dynamic behavior by considering a blockage on the bottom wall, namely, a backward-facing or a forward-facing step (or a combination of both features). Apart from the classical streamwise system aspect ratio $\left(A_{x}\right)$, defined as the cavity length-to-depth ratio $\left(\mathrm{L}_{\mathrm{x}} / \mathrm{d}\right)$, additional non-dimensional parameters required to characterize the problem are, therefore, the ratio $\Gamma=\mathrm{L}_{s} / \mathrm{L}_{\mathrm{x}}$ measuring the horizontal extension of the region with reduced cross-sectional area, $\Lambda=\mathrm{d}_{\mathrm{s}} / \mathrm{d}$, i.e. the nondimensional height or depth of the step (referred to as the "expansion ratio" or "compression ratio") and $\mathrm{A}_{\mathrm{z}}$, i.e. the nondimensional parameter accounting for the extension of the physical domain along the third direction $z$ (namely, the socalled spanwise aspect ratio, required for $3 D$ simulation only and defined as $A_{z}=L_{z} / d$ where $L_{z}$ is the cavity width). 

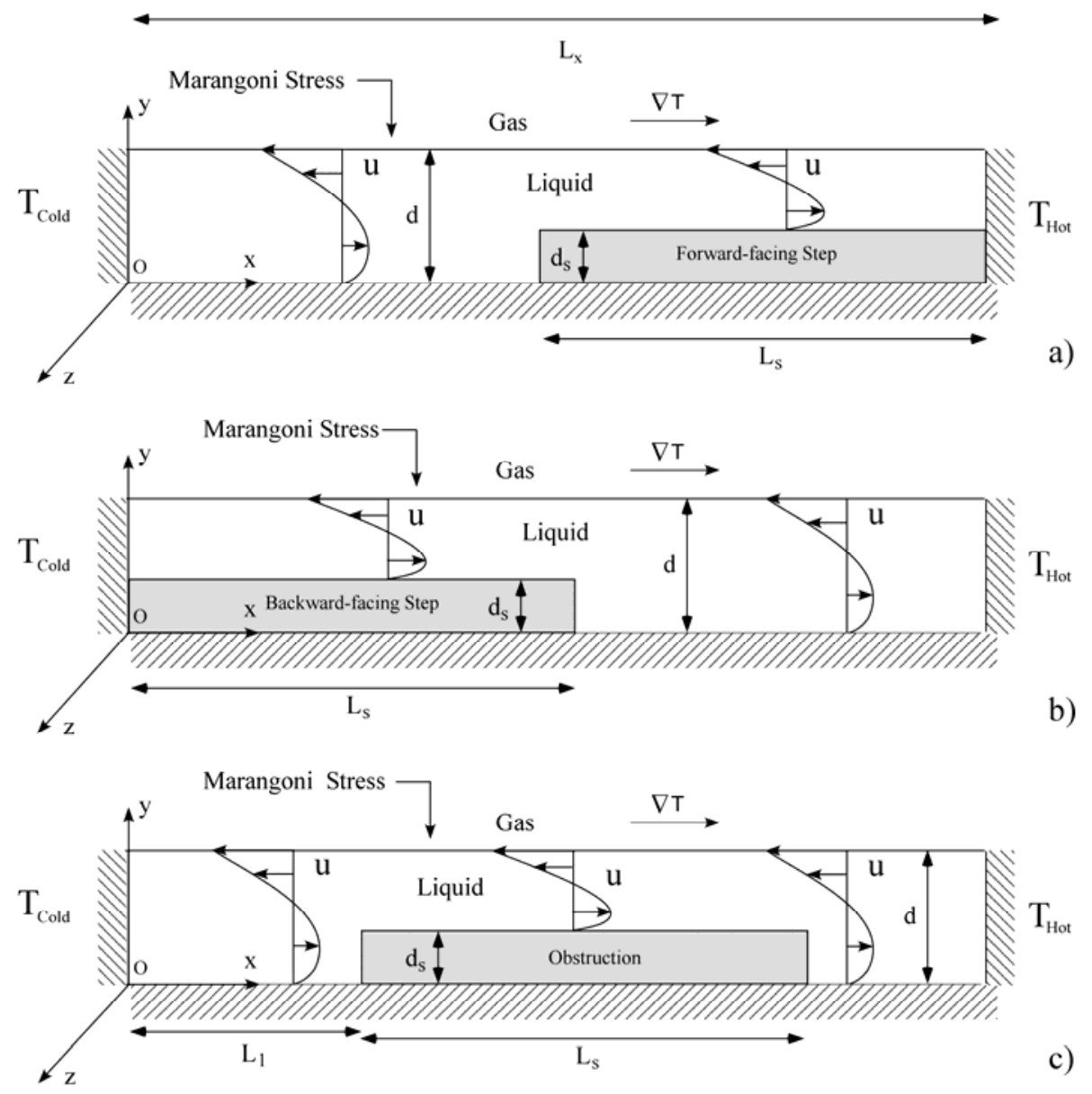

Figure 1: Sketch of the open cavity (so-called R-F configuration) supporting the emergence of hydrothermal disturbances. The cavity can incorporate some geometrical variations.

\subsection{The considered fluid}

As shown by Smith and Davis [14] for an infinite layer, the angle formed by travelling waves with the basic flow behaves as a decreasing function of the Prandtl number (Pr), tending to become very small as $\operatorname{Pr}$ is progressively increased $(\operatorname{Pr}=v / \alpha$ where $v$ and $\alpha$ are the fluid kinematic viscosity and thermal diffusivity, respectively).

Here, the streamwise aspect ratio of the cavity is fixed to $A_{x}=20$ in order to mimic conditions similar to that occurring in a laterally unbounded layer. As work liquid we select the same fluid already considered by Lappa [35], i.e. silicone oil with $\operatorname{Pr}=15$ (see Table I), a rational motivation for such a choice being related to the two-fold requirement to 1) consider a substance for which the departure of the direction of propagation of the hydrothermal waves from that of the imposed temperature gradient is relatively small (the angle formed by travelling waves with the direction of the surface flow is known to be approximately $20^{\circ}$ for a value of the Prandtl number close to 15 ; see, e.g., Burguete et al. [25]; Pelacho et al. [26]) and 2) not to increase excessively the value of the Prandtl number (the latter argument relating to the need to limit the required computational time; such a time being inversely proportional to Pr). 


\begin{tabular}{cc}
\hline \hline Density $\rho\left[\mathrm{gr} / \mathrm{cm}^{3}\right]$ & 0.82 \\
Thermal expansion coefficient $\beta_{\mathrm{T}}[1 / \mathrm{K}]$ & $1.3510^{-3}$ \\
Surface tension $\sigma[$ dyne/cm] & 17.4 \\
$\sigma_{\mathrm{T}}$ [dyne/cmK] & $6.010^{-2}$ \\
Thermal diffusivity $\alpha\left[\mathrm{cm}^{2} / \mathrm{s}\right]$ & $6.4710^{-4}$ \\
Kinematic viscosity $v\left[\mathrm{~cm}^{2} / \mathrm{s}\right]$ & 0.01 \\
Prandtl number & 15 \\
\hline \hline
\end{tabular}

Table I: Physical properties of 1 cSt Silicone oil

\subsection{The balance equations}

Apart from the Prandtl number, the problem is governed by other characteristic numbers, which can be formally introduced by putting the governing (balance) equations for mass, momentum and energy and related boundary conditions in non-dimensional form.

Assuming characteristic thermal scales as reference quantities for velocity and time and scaling all the coordinates with the layer depth $d$, the nondimensional momentum equation can be written as:

$$
\frac{\partial \underline{V}}{\partial t}=-\underline{\nabla} p-\underline{\nabla} \cdot[\underline{V} \underline{V}]+\operatorname{Pr} \nabla^{2} \underline{V}+\operatorname{Pr} R a T \underline{i}_{g}
$$

where $\underline{V}$ and $\mathrm{p}$ are the nondimensional velocity and pressure, respectively and the classical Boussinesq approximation has been implicitly used to account for the production of momentum due to thermal buoyancy. Indeed, Ra is the well-known Rayleigh number defined as $\mathrm{Ra}=\mathrm{g} \beta_{\mathrm{T}} \Delta \mathrm{Td}{ }^{3} / v \alpha$ ( $\beta_{\mathrm{T}}$ being the thermal expansion coefficient and $\Delta \mathrm{T}$ the imposed temperature difference between the hot and cold sidewalls, which implies a linear relationship has been assumed to link density and temperature variations).

With the same scaling units, the continuity and energy equations can be cast in condensed form as:

$\underline{\nabla} \cdot \underline{\mathrm{V}}=0$

and

$\frac{\partial T}{\partial t}=-\underline{\nabla} \cdot[V T]+\nabla^{2} T$

where the nondimensional temperature has been defined as $T=\left(\bar{T}-\bar{T}_{\text {cold }}\right) / \Delta \mathrm{T}$ ( $\bar{T}$ being the dimensional temperature).

In the presence of a free liquid/gas interface, obviously, equations (1)-(3) must be considered together with the so-called Marangoni boundary condition. If viscous stresses in the gas are neglected (in general such assumption is physically consistent because the dynamic viscosity of the 
gas surrounding the free liquid surface is much smaller than the viscosity of the considered liquid) such condition reads:

$$
\frac{\partial \underline{V}_{S}}{\partial n}=-M a \underline{\nabla}_{S} T
$$

where $\underline{\mathrm{n}}$ is the direction perpendicular to the free interface (planar in our case), $\underline{\nabla}_{S}$ the derivative tangential to the interface (the $\mathrm{x}$ direction) and $\underline{V}_{\mathrm{s}}$ the surface velocity vector. Moreover, Ma is the Marangoni number defined as $\mathrm{Ma}=\sigma_{\mathrm{T}} \Delta \mathrm{Td}^{3} / \mu \alpha$ (where $\sigma_{\mathrm{T}}$ is the surface tension derivative with respect to temperature and $\mu=v \rho$, $\rho$ being the liquid density).

\subsection{The Initial and Boundary conditions}

As initial conditions we consider:

$\mathrm{t}=0: \quad \underline{V}(\mathrm{x}, \mathrm{y}, \mathrm{z})=0, \mathrm{~T}(\mathrm{x}, \mathrm{y}, \mathrm{z})=\mathrm{x} / \mathrm{A}_{\mathrm{x}}$

where $\underline{V}=0$ implies $\mathrm{u}=\mathrm{v}=\mathrm{w}=0(\mathrm{u}, \mathrm{v}$ and $\mathrm{w}$ being the velocity components along the $\mathrm{x}, \mathrm{y}$ and $\mathrm{z}$ directions, respectively), i.e. the liquid is motionless with a linear temperature profile along the $\mathrm{x}$ coordinate (the temperature is $\mathrm{T}_{\text {Cold }}=0$ on the cold sidewall and $\mathrm{T}_{\text {Hot }}=1$ on the other side). Adiabatic conditions are assumed for the free liquid-gas interface, while the bottom wall can feature both adiabatic and conducting conditions. Accordingly, the boundary conditions can be cast in compact form as:

$\mathrm{u}=0, \mathrm{v}=0, \mathrm{w}=0$ and $\mathrm{T}=0$ at $\mathrm{x}=0$ (left wall)

$\mathrm{u}=0, \mathrm{v}=0, \mathrm{w}=0$ and $\mathrm{T}=1$ at $\mathrm{x}=\mathrm{A}_{\mathrm{x}}$ (right wall)

$\mathrm{v}=0, \partial \mathrm{u} / \partial \mathrm{y}=-\mathrm{Ma} \partial \mathrm{T} / \partial \mathrm{x}, \partial \mathrm{w} / \partial \mathrm{y}=-\mathrm{Ma} \partial \mathrm{T} / \partial \mathrm{z}$ and $\partial \mathrm{T} / \partial \mathrm{y}=0$ at $\mathrm{y}=1$ (free surface)

Equation (8) enforces a flow by tangential variation of the surface tension. Obviously, purely stressfree conditions, that is pure buoyancy flow, can be recovered by simply setting $\mathrm{Ma}=0$.

As already outlined in the introduction, an additional useful non-dimensional parameter measuring the relative importance of surface-tension driven and buoyancy effects can be introduced as:

$\frac{M a}{R a}=W=\frac{\sigma_{T}}{g \rho \beta_{T}} d^{-2}$

$W=B_{d}{ }^{-1}$

where $B_{d}$ is the well-known dynamic Bond number. 
The sidewalls along the $\mathrm{z}$ direction (at $\mathrm{z}=0$ and $\mathrm{z}=\mathrm{A}_{\mathrm{z}}$ ) are assumed to be adiabatic as well. Accordingly, the following conditions apply:

$\mathrm{u}=0, \mathrm{v}=0, \mathrm{w}=0, \partial \mathrm{T} / \partial \mathrm{z}=0$ at $\mathrm{z}=0$

$\mathrm{u}=0, \mathrm{v}=0, \mathrm{w}=0, \partial \mathrm{T} / \partial \mathrm{z}=0$ at $\mathrm{z}=\mathrm{A}_{\mathrm{z}}$

For the configuration with the backward-facing step, the additional boundary conditions of both kinematic and thermal nature to be taken into account read:

$\mathrm{u}=0, \mathrm{v}=0, \mathrm{w}=0$ at $\mathrm{y}=\Lambda$ for $0 \leq \mathrm{x} \leq \mathrm{A}_{\mathrm{x}} \Gamma$

$\mathrm{u}=0, \mathrm{v}=0, \mathrm{w}=0$ at $\mathrm{x}=\mathrm{A}_{\mathrm{x}} \Gamma$ for $0 \leq \mathrm{y} \leq \Lambda$

$\mathrm{u}=0, \mathrm{v}=0, \mathrm{w}=0$ at $\mathrm{y}=0$ for $\mathrm{A}_{\mathrm{x}}(1-\Gamma) \leq \mathrm{x} \leq \mathrm{A}_{\mathrm{x}}$

and (adiabatic bottom wall)

$\partial \mathrm{T} / \partial \mathrm{y}=0$ at $\mathrm{y}=\Lambda$ for $0 \leq \mathrm{x} \leq \mathrm{A}_{\mathrm{x}} \Gamma$

$\partial \mathrm{T} / \partial \mathrm{x}=0$ at $\mathrm{x}=\mathrm{A}_{\mathrm{x}} \Gamma$ for $0 \leq \mathrm{y} \leq \Lambda$

$\partial \mathrm{T} / \partial \mathrm{y}=0$ at $\mathrm{y}=0$ for $\mathrm{A}_{\mathrm{x}}(1-\Gamma) \leq \mathrm{x} \leq \mathrm{A}_{\mathrm{x}}$

or (for the case of conducting bottom)

$\mathrm{T}=\mathrm{x} / \mathrm{A}_{\mathrm{x}}$ at $\mathrm{y}=\Lambda$ for $0 \leq \mathrm{x} \leq \mathrm{A}_{\mathrm{x}} \Gamma$

$\mathrm{T}=\Gamma$ at $\mathrm{x}=\mathrm{A}_{\mathrm{x}} \Gamma$ for $0 \leq \mathrm{y} \leq \Lambda$

$\mathrm{T}=\mathrm{x} / \mathrm{A}_{\mathrm{x}}$ at $\mathrm{y}=0$ for $\mathrm{A}_{\mathrm{x}}(1-\Gamma) \leq \mathrm{x} \leq \mathrm{A}_{\mathrm{x}}$

For the configuration with the forward-facing step:

$\mathrm{u}=0, \mathrm{v}=0, \mathrm{w}=0$ at $\mathrm{y}=0$ for $0 \leq \mathrm{x} \leq \mathrm{A}_{\mathrm{x}}(1-\Gamma)$

$\mathrm{u}=0, \mathrm{v}=0, \mathrm{w}=0$ at $\mathrm{x}=\mathrm{A}_{\mathrm{x}}(1-\Gamma)$ for $0 \leq \mathrm{y} \leq \Lambda$

$\mathrm{u}=0, \mathrm{v}=0, \mathrm{w}=0$ at $\mathrm{y}=\Lambda$ for $\mathrm{A}_{\mathrm{x}}(1-\Gamma) \leq \mathrm{x} \leq \mathrm{A}_{\mathrm{x}}$

and (adiabatic bottom wall)

$\partial \mathrm{T} / \partial \mathrm{y}=0$ at $\mathrm{y}=0$ for $0 \leq \mathrm{x} \leq \mathrm{A}_{\mathrm{x}}(1-\Gamma)$

$\partial \mathrm{T} / \partial \mathrm{x}=0$ at $\mathrm{x}=\mathrm{A}_{\mathrm{x}}(1-\Gamma)$ for $0 \leq \mathrm{y} \leq \Lambda$

$\partial \mathrm{T} / \partial \mathrm{y}=0$ at $\mathrm{y}=\Lambda$ for $\mathrm{A}_{\mathrm{x}}(1-\Gamma) \leq \mathrm{x} \leq \mathrm{A}_{\mathrm{x}}$

or (for the case of conducting bottom)

$\mathrm{T}=\mathrm{x} / \mathrm{A}_{\mathrm{x}}$ at $\mathrm{y}=0$ for $0 \leq \mathrm{x} \leq \mathrm{A}_{\mathrm{x}}(1-\Gamma)$

$\mathrm{T}=(1-\Gamma)$ at $\mathrm{x}=\mathrm{A}_{\mathrm{x}}(1-\Gamma)$ for $0 \leq \mathrm{y} \leq \Lambda$

$\mathrm{T}=\mathrm{x} / \mathrm{A}_{\mathrm{x}}$ at $\mathrm{y}=\Lambda$ for $\mathrm{A}_{\mathrm{x}}(1-\Gamma) \leq \mathrm{x} \leq \mathrm{A}_{\mathrm{x}}$

For the configuration with the obstruction in the center: 
$\mathrm{u}=0, \mathrm{v}=0, \mathrm{w}=0$ at $\mathrm{y}=0$ for $0 \leq \mathrm{x} \leq \mathrm{A}_{\mathrm{x}} \Gamma_{1}$

$\mathrm{u}=0, \mathrm{v}=0, \mathrm{w}=0$ at $\mathrm{x}=\mathrm{A}_{\mathrm{x}} \Gamma_{1}$ for $0 \leq \mathrm{y} \leq \Lambda$

$\mathrm{u}=0, \mathrm{v}=0, \mathrm{w}=0$ at $\mathrm{y}=\Lambda$ for $\mathrm{A}_{\mathrm{x}} \Gamma_{1} \leq \mathrm{x} \leq \mathrm{A}_{\mathrm{x}}\left(\Gamma+\Gamma_{1}\right)$

$\mathrm{u}=0, \mathrm{v}=0, \mathrm{w}=0$ at $\mathrm{x}=\mathrm{A}_{\mathrm{x}}\left(\Gamma+\Gamma_{1}\right)$ for $0 \leq \mathrm{y} \leq \Lambda$

$\mathrm{u}=0, \mathrm{v}=0, \mathrm{w}=0$ at $\mathrm{y}=0$ for $\mathrm{A}_{\mathrm{x}}\left(\Gamma+\Gamma_{1}\right) \leq \mathrm{x} \leq \mathrm{A}_{\mathrm{x}}$

and (adiabatic bottom wall)

$\partial \mathrm{T} / \partial \mathrm{y}=0$ at $\mathrm{y}=0$ for $0 \leq \mathrm{x} \leq \mathrm{A}_{\mathrm{x}} \Gamma_{1}$

$\partial \mathrm{T} / \partial \mathrm{x}=0$ at $\mathrm{x}=\mathrm{A}_{\mathrm{x}} \Gamma_{1}$ for $0 \leq \mathrm{y} \leq \Lambda$

$\partial \mathrm{T} / \partial \mathrm{y}=0$ at $\mathrm{y}=\Lambda$ for $\mathrm{A}_{\mathrm{x}} \Gamma_{1} \leq \mathrm{x} \leq \mathrm{A}_{\mathrm{x}}\left(\Gamma+\Gamma_{1}\right)$

$\partial \mathrm{T} / \partial \mathrm{x}=0$ at $\mathrm{x}=\mathrm{A}_{\mathrm{x}}\left(\Gamma+\Gamma_{1}\right)$ for $0 \leq \mathrm{y} \leq \Lambda$

$\partial \mathrm{T} / \partial \mathrm{y}=0$ at $\mathrm{y}=0$ for $\mathrm{A}_{\mathrm{x}}\left(\Gamma+\Gamma_{1}\right) \leq \mathrm{x} \leq \mathrm{A}_{\mathrm{x}}$

or (for the case of conducting bottom)

$\mathrm{T}=\mathrm{x} / \mathrm{A}_{\mathrm{x}}$ at $\mathrm{y}=0$ for $0 \leq \mathrm{x} \leq \mathrm{A}_{\mathrm{x}} \Gamma_{1}$

$\mathrm{T}=\Gamma_{1}$ at $\mathrm{x}=\mathrm{A}_{\mathrm{x}} \Gamma_{1}$ for $0 \leq \mathrm{y} \leq \Lambda$

$\mathrm{T}=\mathrm{x} / \mathrm{A}_{\mathrm{x}}$ at $\mathrm{y}=\Lambda$ for $\mathrm{A}_{\mathrm{x}} \Gamma_{1} \leq \mathrm{x} \leq \mathrm{A}_{\mathrm{x}}\left(\Gamma+\Gamma_{1}\right)$

$\mathrm{T}=\left(\Gamma+\Gamma_{1}\right)$ at $\mathrm{x}=\mathrm{A}_{\mathrm{x}}\left(\Gamma+\Gamma_{1}\right)$ for $0 \leq \mathrm{y} \leq \Lambda$

$\mathrm{T}=\mathrm{x} / \mathrm{A}_{\mathrm{x}}$ at $\mathrm{y}=0$ for $\mathrm{A}_{\mathrm{x}}\left(\Gamma+\Gamma_{1}\right) \leq \mathrm{x} \leq \mathrm{A}_{\mathrm{x}}$

As already explained to a certain extent in the introduction, our choice to use adiabatic or conducting thermal conditions for the bottom wall follows a common practice in the literature (essentially relying on the paradigm of a series of purely conceptual boundary conditions). The former type of condition would imply the use of materials with negligible thermal conductivity (being exactly zero from a theoretical standpoint, corresponding to plastics or other insulating materials in reality); the latter might be approached in practice using materials with extremely high thermal conductivity such as copper, silver or gold. For the sake of completeness, we wish to emphasize that, for the cases considered here (featuring a sudden thickening of the wall, i.e. the obstruction), even with such materials (plastics or highly conducting metals) a thermal perturbation would appear in correspondence of the step causing a departure from the abovementioned idealized conditions. The results presented in the following sections for the cases with adiabatic or conducting wall must therefore be considered valid in the mathematical limit as the wall thermal conductivity is zero or infinite, respectively.

\subsection{The Numerical method}

The method we have used for the solution of the governing equations for incompressible fluid flow (cast in compact form in Sect. 2.3 with the related initial and boundary conditions reported in detail in Sect. 2.4 for all the different configurations of interest) may be regarded as a classical 
implementation of the strategy originally conceived by Harlow and Welch [42], Chorin [43] and Temam [44] on the basis of the well-known Ladyzhenskaya decomposition theorem (see, e.g., Ladyzhenskaya [45]), by which any vector function can always be decomposed into a divergencefree part $\underline{\mathrm{V}}$ and the gradient of a scalar potential -P (a curl-free part). The resulting numerical method is indeed articulated in two main steps by which an intermediate velocity field is initially determined, which is then corrected in a second stage implemented to guarantee that the resulting velocity field is "unique” on the basis of the theorem and satisfies all the overarching equations at the same time.

More precisely, in the first stage a simplified version of the momentum equation is solved in which the pressure term is not taken into account. Similarly, the constraint represented by the continuity equation is not accounted for in this stage. The vector field determined in this way does not satisfy the incompressibility requirement. Nevertheless, it has the correct content of vorticity as the pressure gradient does not contribute to such content. In a second stage, this field is expressly corrected to make the flow solenoidal. The velocity field is indeed re-processed numerically by forcing it to satisfy an additional equation for the pressure obtained by combining the complete momentum equation and the continuity equation.

Though these algorithms have long been used to determine steady-state solutions, the major advantage they offer is related to their stability, flexibility and reliability in providing meaningful information about time-dependent dynamics, especially with regard to their ability to capture bifurcations and instability phenomena.

As a natural consequence of the nature of these strategies (generally presented in textbooks as 'projection', 'fractional-step' or 'pressure-correction' based methods), some special care must be devoted to make sure that the fundamental primitive variables involved in the process (namely pressure and velocity, which are computed 'separately' by virtue of the peculiar approach described before), are properly coupled. Ill-resolved coupling can indeed result in the undesired onset of spurious oscillations (Choi et al. [46, 47]). This typically leads to the need for the so-called staggered-variables arrangement, by which pressure and velocity do not occupy the same positions on the computational grid.

In the light of the above arguments, the balance equations (eqs. 1-3) and related boundary conditions (eqs. 6-17) have been solved numerically by a time-explicit finite-difference method (two-step primitive-variable approach as illustrated above) relying on a structured grid with staggered collocation of the fluid-dynamic variables. In particular, we have used forward differences in time and Lax-Wendroff (central-differencing) schemes in space (with second order accuracy) to discretize the pertinent terms in the energy and momentum equations. As this numerical framework is a standard one and it has already enjoyed a widespread success in the literature, the interested reader is referred to the existing literature on the subject for an exhaustive treatment.

Prior to expanding on the numerical results, anyhow, it is worth mentioning that the code used for the present analysis was validated through comparison with relevant numerical results in the 
literature, namely, those presented by Shevtsova et al. [6] for the case of mixed thermocapillarythermogravitational convection. These authors examined a situation akin to that addressed here (A=24.7, $\quad \operatorname{Pr}=13.9$, adiabatic liquid-gas interface). For $\mathrm{Ma}=18154.5$ and $\mathrm{Ra}=2578$ $\left(\mathrm{Bo}_{\mathrm{dyn}}=\mathrm{Ra} / \mathrm{Ma}=0.142\right.$, Grid $\left.600 \times 100\right)$, these authors could clearly identify the frequency of the emerging traveling wave $\left(\Omega_{\mathrm{HTW}}=45.28\right)$. For the same conditions and a uniform mesh $600 \mathrm{x} 30$ (that is 30 grid points per unit length), we could obtain a frequency 46.03 (that is, agreement lying within 2\%). According to a grid-refinement study (not included here for the sake of brevity) we deemed the above-mentioned requirement (relating to the use of a minimum of 30 grid points per unit length in every direction) to be effective for the conditions considered in the present study as well (leading to a total of $\cong 8 \times 10^{6}$ grid points for the 3D cases). The interested reader is referred to Lappa [48] for general information about our criteria for the selection of the spatial and temporal resolution, or to the dedicated tables in Refs [35] and [49] for specific data about the sensitivity of the numerically simulated Marangoni convection to the mesh for $\operatorname{Pr}=15, \mathrm{~A}=20$ and $\mathrm{Ma}=3 \times 10^{4}$ and $\mathrm{Ma}=2 \times 10^{4}$, respectively.

\section{Results}

In order to extend the earlier results by [35] for pure Marangoni flow (no buoyancy), the following conditions have been considered: $\mathrm{Ma}=2 \times 10^{4}, \mathrm{~A}_{\mathrm{X}}=20, \Gamma=0.5, \Gamma_{1}=0.25$ (where applicable) and $0.25 \leq \Lambda \leq 0.45$. As outlined in the introduction, here we address the influence of thermal buoyancy on Marangoni flow and related hydrothermal waves in the presence of a blockage on the bottom (to change the local depth of the fluid), placing it at both upstream and downstream locations or in the center. Moreover, the response of the system is explored with respect to either adiabatic or conducting wall because, as illustrated in the introduction, these thermal boundary conditions are expected to play an appreciable role on the emerging dynamics together with the variations in the shape of the container.

The reader may get a first glimpse into the patterning behavior of such a system by taking a look at Fig. 2, where snapshots of the "typical" temperature field and flow structure produced by twodimensional simulations have been reported in an ordered fashion for different configurations (for a fixed non-dimensional time and a fixed value of the Rayleigh number by assuming $\Lambda=0.25$, $\mathrm{Ra}=1000$ and varying the geometry and the thermal boundary conditions at the bottom wall). Such snapshots may be regarded as a first example of dynamics for conditions in which the emerging flow is completely dominated by the presence of hydrothermal waves.

The flow itself looks as a series of rolls spreading in the upstream direction with variable size, intensity and spacing according to the region where they are temporarily located (the size of rolls becomes smaller while their number increases in the portion of the cavity with reduced crosssectional area).

The impact of the thermal boundary conditions at the bottom wall can be seen essentially in the topology of the isotherms, which changes especially in proximity to the hot wall. As a result, the 
fluid located close to the right side of the cavity tends to be appreciably hotter when the bottom wall is assumed to be conducting. Some differences can also be seen in the intensity of such rolls, which is appreciably higher in this case.
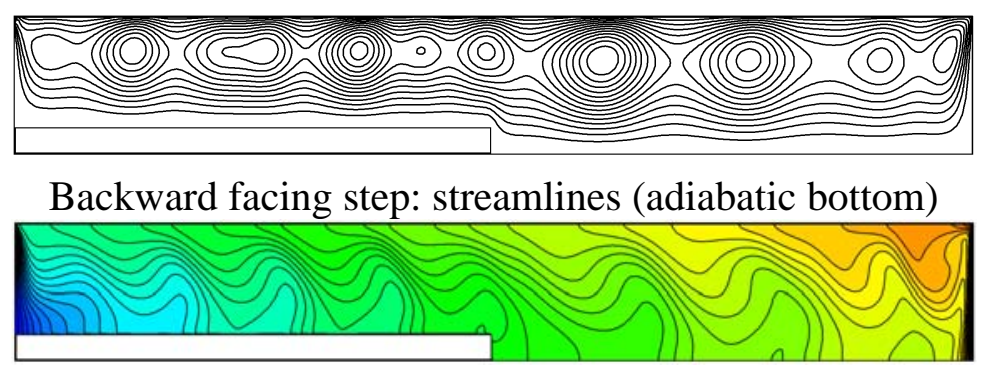

Backward facing step: temperature field (adiabatic bottom)

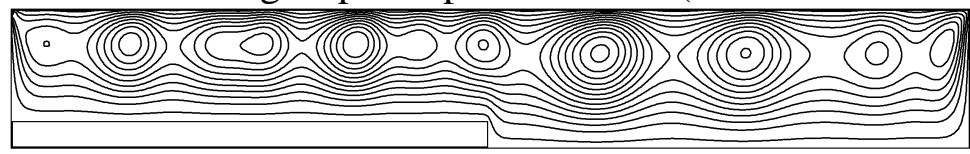

Backward facing step: streamlines (conducting bottom)

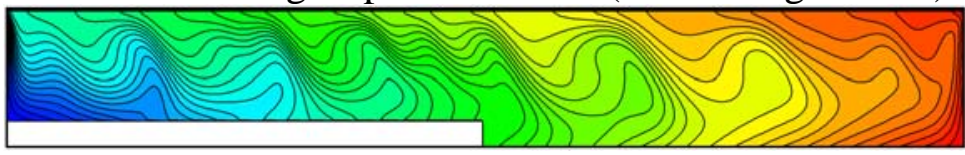

Backward facing step: temperature field (conducting bottom)

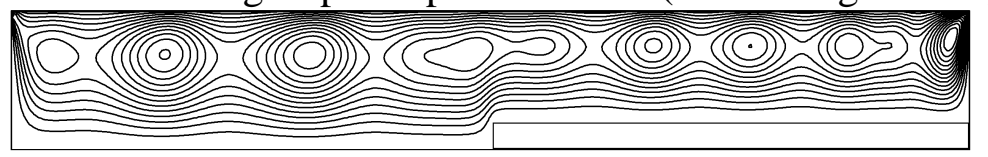

Forward facing step: streamlines (adiabatic bottom)

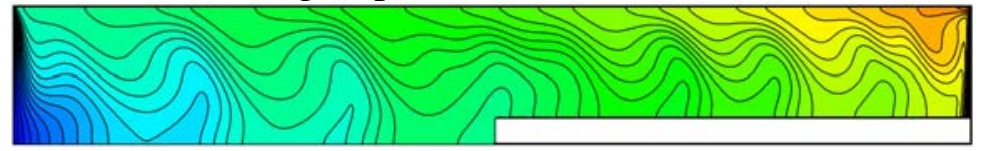

Forward facing step: temperature field (adiabatic bottom)

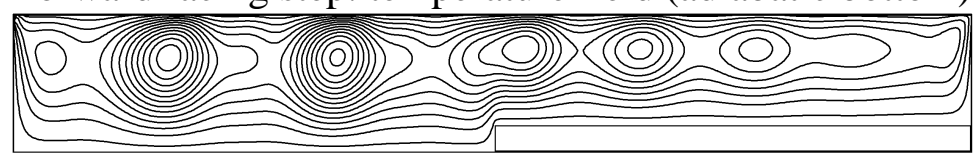

Forward facing step: streamlines (conducting bottom)

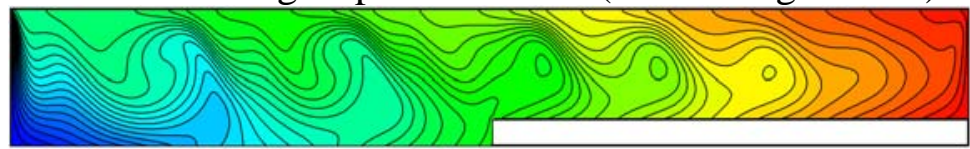

Forward facing step: temperature field (conducting bottom)

Figure 2: Snapshots of streamlines distribution and temperature field for different geometrical configurations (backward-facing and forward-facing steps) and thermal boundary conditions at the bottom wall $\left(\Lambda=0.25, \mathrm{Ma}=2 \times 10^{4}, \mathrm{Ra}=10^{3}, 2 \mathrm{D}\right.$ simulations, $\left.\mathrm{t}=3\right)$. 

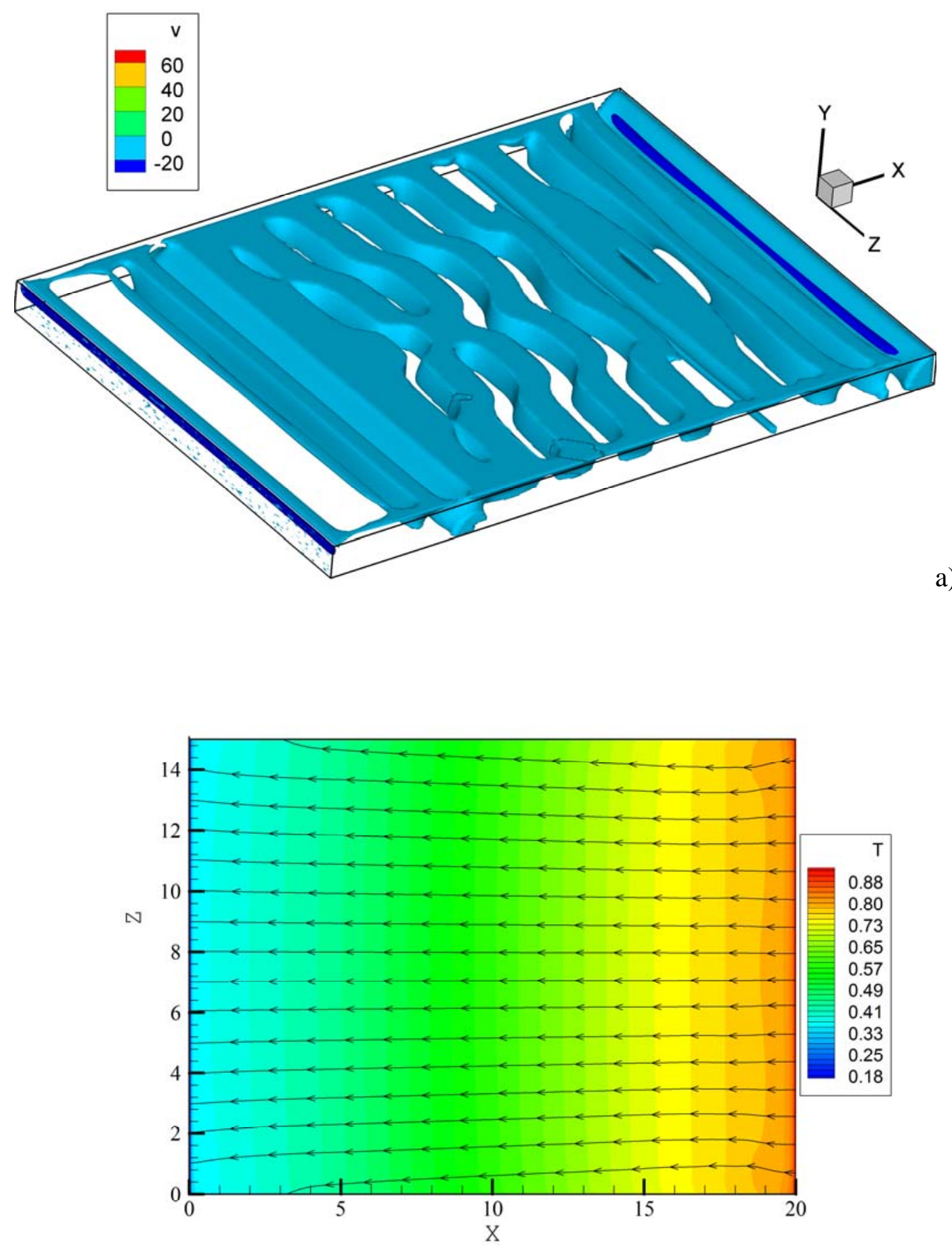

b) 


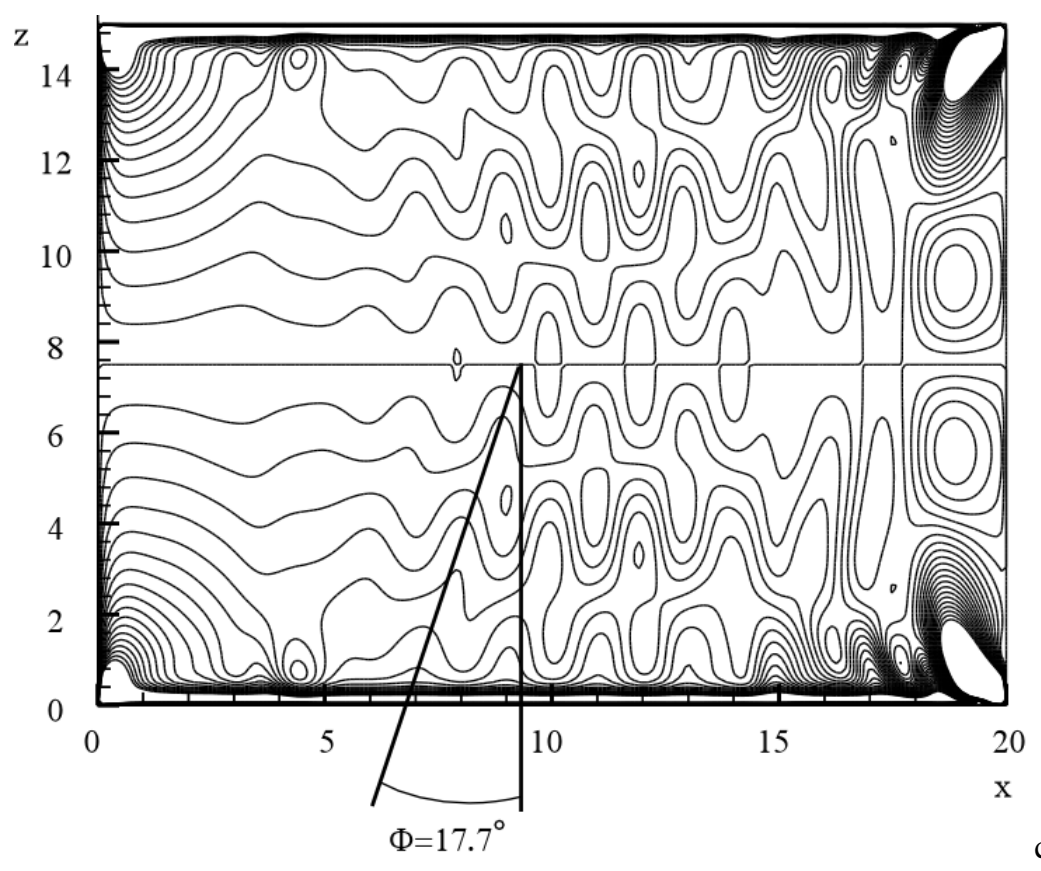

Figure 3: Structure of the thermofluid-dynamic field in a 3D layer with central obstruction, adiabatic conditions on the bottom wall, isothermal walls at $x=0$ and $x=20$, adiabatic surface and adiabatic walls at $\mathrm{z}=0$ and $\mathrm{z}=15\left(\mathrm{~A}_{\mathrm{x}}=20, \mathrm{~A}_{\mathrm{z}}=15, \mathrm{Ma}=2 \times 10^{4}, \mathrm{Ra}=10^{3}, 3 \mathrm{D}\right.$ simulations with $\cong 8 \times 10^{6}$ grid points, $t=2$ ): a) isosurfaces (snapshot) of the vertical component of velocity revealing the presence of transverse rolls with slightly distorted shapes, which travel from the cold side towards the hot side, b) snapshot of surface temperature field, c) distribution of the longitudinal component of velocity $(\mathrm{w})$ on the free interface revealing the inclination angle of travelling waves with respect to the basic flow.

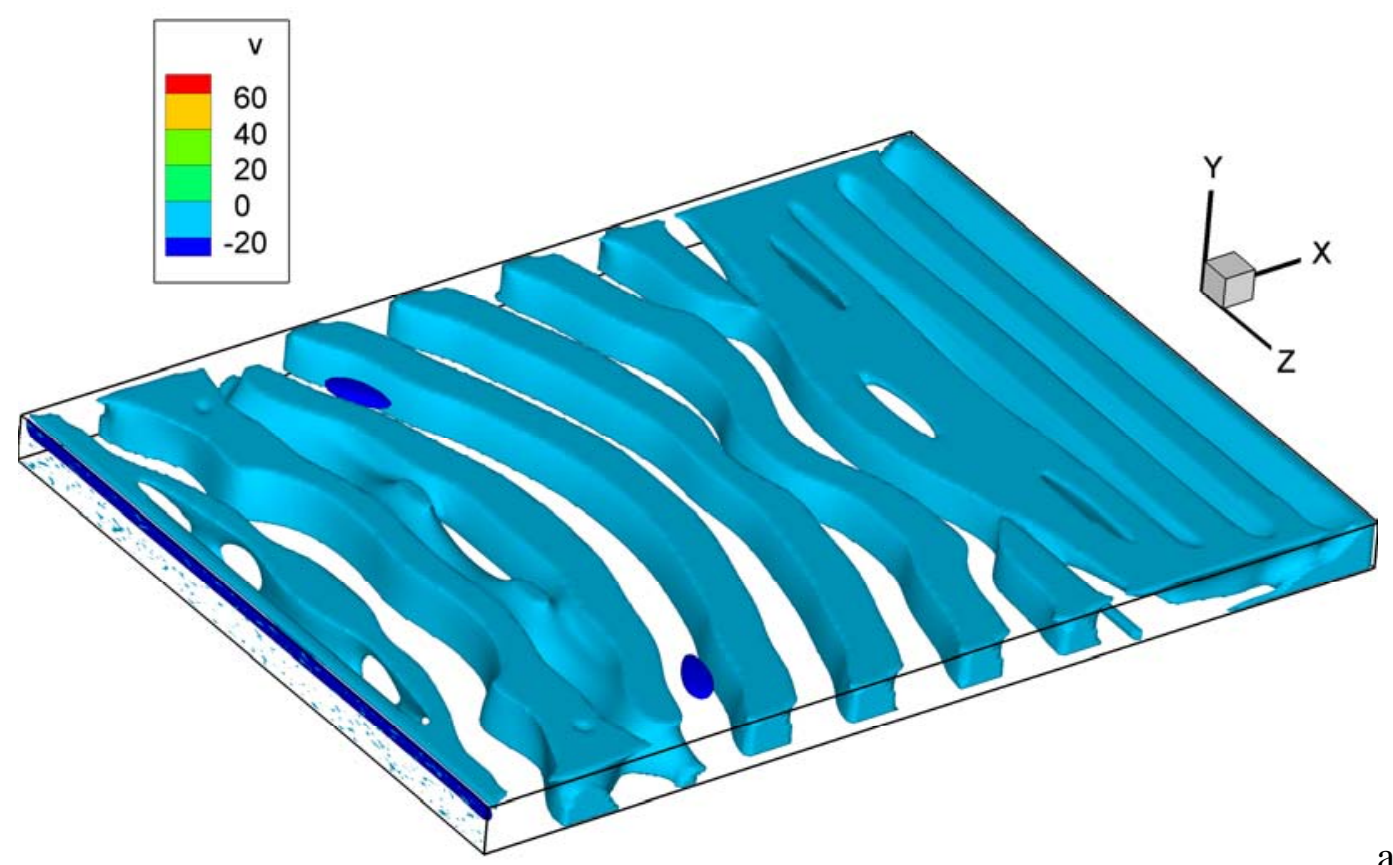

a) 

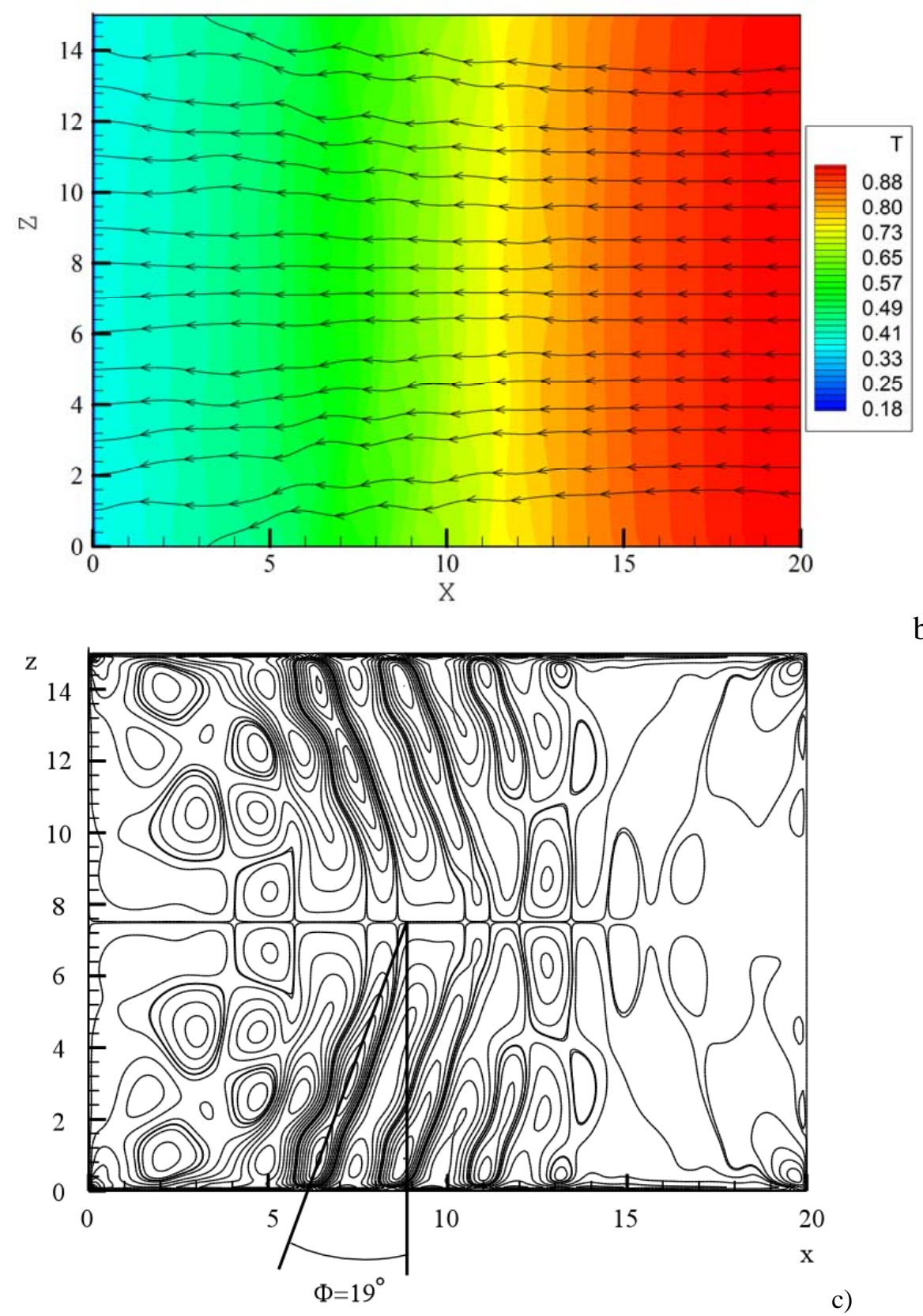

b)

Figure 4: Structure of the thermofluid-dynamic field in a 3D layer with central obstruction, conducting conditions on the bottom wall, isothermal walls at $\mathrm{x}=0$ and $\mathrm{x}=20$, adiabatic surface and adiabatic walls at $\mathrm{z}=0$ and $\mathrm{z}=15\left(\mathrm{~A}_{\mathrm{x}}=20, \mathrm{~A}_{\mathrm{z}}=15, \mathrm{Ma}=2 \times 10^{4}, \mathrm{Ra}=10^{3}, 3 \mathrm{D}\right.$ simulations, $\left.\mathrm{t}=2\right)$ : a) isosurfaces (snapshot) of the vertical component of velocity revealing the presence of transverse rolls with slightly distorted shapes, which travel from the cold side towards the hot side, b) snapshot of surface temperature field, c) distribution of the longitudinal component of velocity (w) on the free interface revealing the inclination angle of travelling waves with respect to the basic flow.

Three-dimensional effects being related to these flows can be seen in Figs. 3 and 4. These figures illustrate that apart from a component in the streamwise (transverse) direction, as expected, HTWs also display components in the spanwise (longitudinal) direction. 
Initially two waves are produced with opposite components along $\mathrm{z}$ and equal amplitude. The superposition of such waves is responsible for the z-symmetric pattern visible in both Figs. 3 and 4. The most interesting (and useful) outcome of these figures, however, is the clear indication they provide about the possibility to rely on two-dimensional (2D) simulations for further (parametric) investigation of such dynamics. Indeed, in agreement with the prediction of existing linear stability analyses, the inclination angle of such waves is relatively small $\left(\Phi \cong 18^{\circ}\right.$ and $19^{\circ}$ for the present cases with adiabatic and conducting bottom walls, respectively), which may be regarded as a clear hint for relying on a strategy based on two-dimensional (2D) simulations to explore (with an acceptable level of approximation) the system response over a large range of conditions and parameters (accordingly, 2D results are discussed in Sects. 3.1 and 3.2, while 3D simulations are expressly re-introduced in Sect. 3.3 to assess the "validity" of this modus operandi).

\subsection{The Hierarchy of spatio-temporal behaviors}

A deeper analysis of the problem requires a detailed description of the hierarchy of spatio-temporal behaviors taken by the system as the Rayleigh number is increased while keeping fixed the Marangoni number. Leaving aside for a while the thermal boundary condition at the bottom wall, additional branches of such a tree are obviously produced by the other degrees of freedom involved in the considered problem, namely, the location of the obstruction and its "thickness".

In order to fix the ideas, as 'representative' scenario for such dynamics in the following we focus on the case relating to the cavity with the central obstruction subjected to increasing values of Ra for a fixed (not too high) height of the step (e.g., the same value already considered in Fig. 2, i.e. $\Lambda=0.25)$.
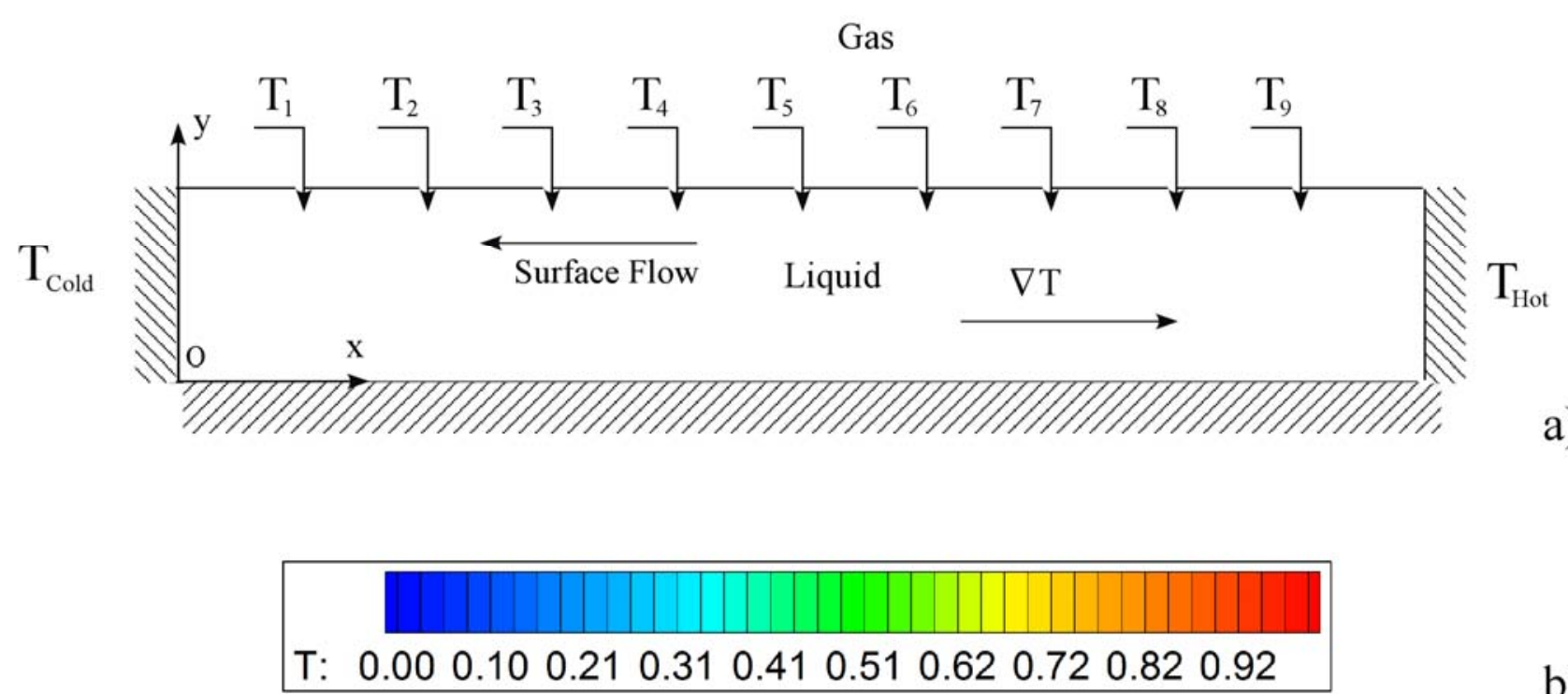

Figure 5: a) Distribution of numerical probes along the cavity, (b) contour legend related to temperature maps. 
In such a context, we show that a "signal-aided" analysis can provide instructive information on the evolution taken by the travelling wave. By 'signals', we mean 'numerically probed' temperatures at fixed points along the free liquid-gas interface ('numerical thermocouples' aligned and evenly spaced with respect to the horizontal direction, as sketched in Fig. 5).

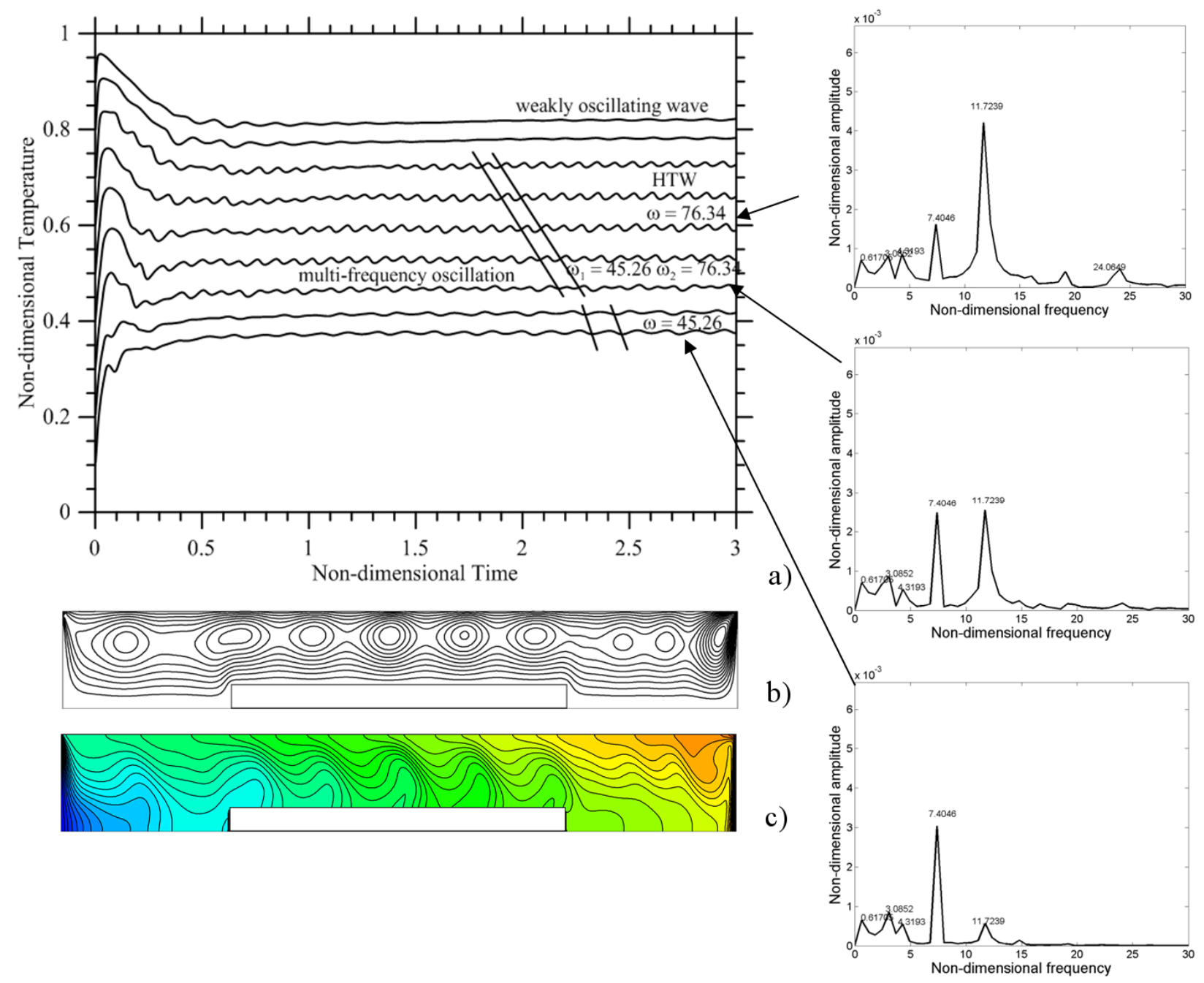

Figure 6: $\left(\operatorname{Pr}=15, \mathrm{~A}_{\mathrm{x}}=20\right.$, central obstruction, $\Gamma_{1}=0.25, \Lambda=0.25, \mathrm{Ma}=2.0 \times 10^{4}, \mathrm{Ra}=10^{3}$, adiabatic bottom, 2D simulations): a) plot of signals provided by numerical probes with related frequency spectra, b) snapshot of streamlines and c) temperature field at $t=3$.

Along these lines, Fig. 6 shows the distribution of temperature signals as a function of time and space for the same conditions considered in Fig. 2, i.e., $\mathrm{Ra}=1000$ and adiabatic bottom wall. As witnessed by this figure, for such case the oscillatory signals display a continuous phase shift along the $\mathrm{x}$-coordinate in the central part of the cavity (made evident in the plot by the inclined solid lines added to it to track the progressive displacement of a period of oscillation), which may be regarded as the "typical signature of a HTW". 
Interestingly, the slope of these lines is different in the post-obstruction region, which indicates that the wave undergoes a decrease in velocity in that area. Other differences can be identified in the signal frequency spectrum. The single frequency state in the pre- or post-obstruction regions $\left(\mathrm{x}<\mathrm{A}_{\mathrm{x}} / 4\right.$ or $\left.\mathrm{x}>3 \mathrm{~A}_{\mathrm{x}} / 4\right)$ is taken over by a two-frequency spectrum in the central region with nondimensional angular frequencies $\omega_{1}=45.26$ and $\omega_{2}=76.34$.

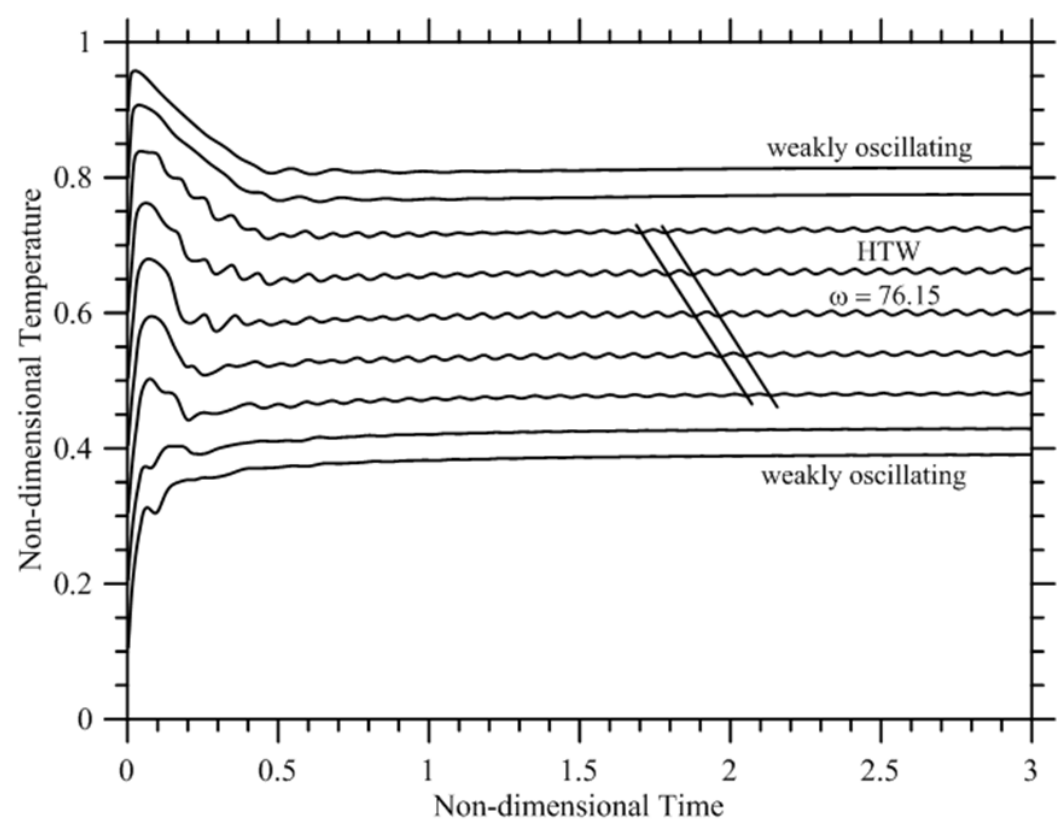

a)

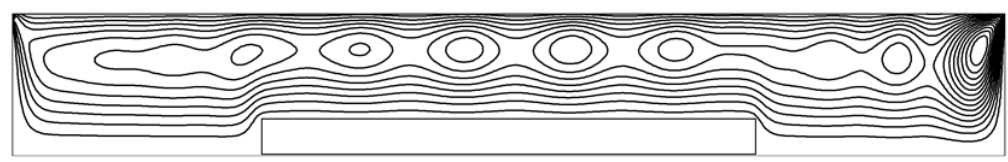

b)

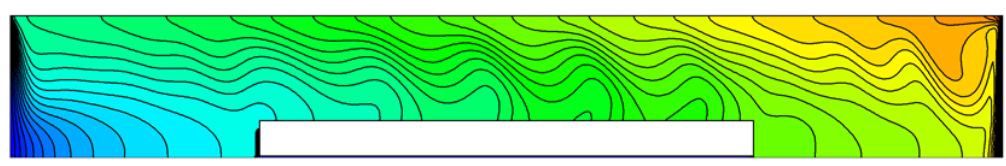

c)

Figure 7: $\left(\operatorname{Pr}=15, \mathrm{~A}_{\mathrm{x}}=20\right.$, central obstruction, $\Gamma_{1}=0.25, \Lambda=0.25, \mathrm{Ma}=2.0 \times 10^{4}, \mathrm{Ra}=3 \times 10^{3}$, adiabatic bottom, 2D simulations): a) plot of signals provided by numerical probes, b) snapshot of streamlines and c) temperature field at $\mathrm{t}=3$.

These two frequencies reflect the physical phenomena at play in the considered system, namely a disturbance travelling continuously from the cold wall towards the right wall with constant angular velocity $\omega_{1}$ (which would correspond to the classical streamwise component of a wave emerging in an unobstructed layer) and the resulting modulation due to the forcing through the region of reduced cross-sectional area (leading locally to an acceleration of the wave, i.e. $\omega_{2}$ ).

The evolution of such a system for increasing values of the Rayleigh number is shown in Figs. 7 and 8 , from which the reader will immediately realize that buoyancy exerts its expected stabilizing role on the HTW essentially via a two-stage process. 


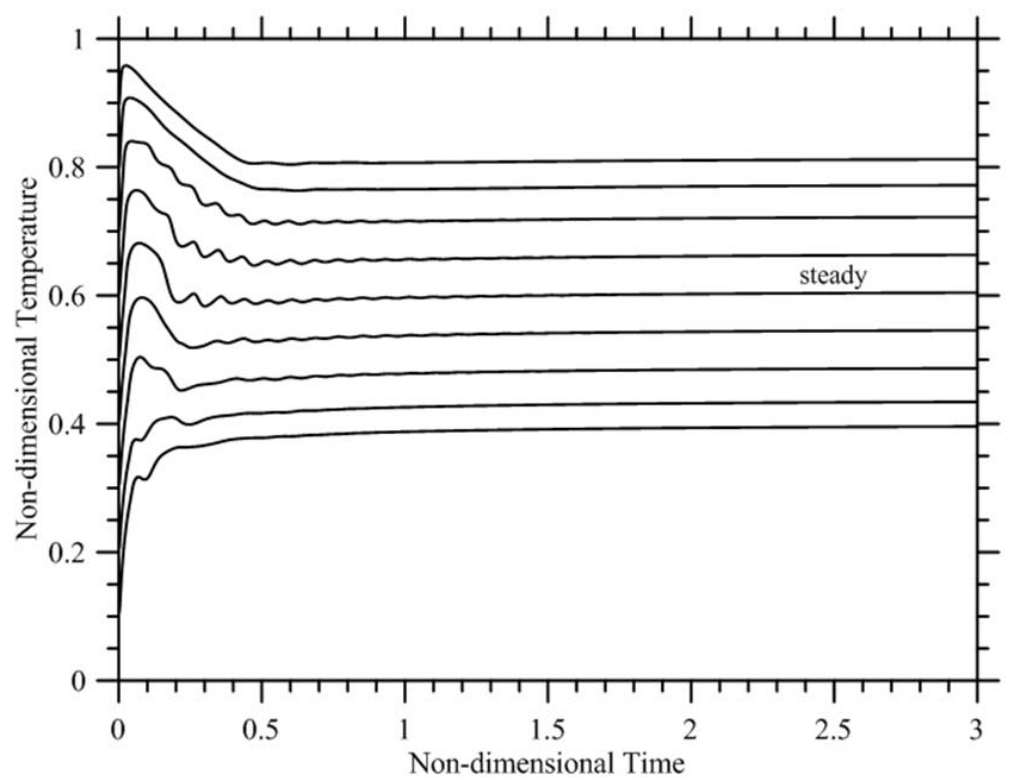

a)

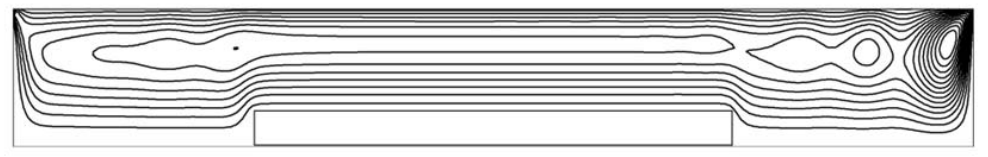

b)

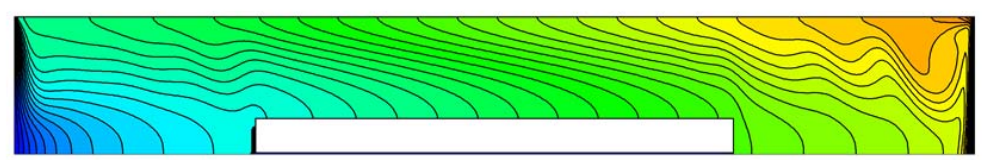

c)

Figure 8: $\left(\operatorname{Pr}=15, \mathrm{~A}_{\mathrm{x}}=20\right.$, central obstruction, $\Gamma_{1}=0.25, \Lambda=0.25, \mathrm{Ma}=2.0 \times 10^{4}, \mathrm{Ra}=5 \times 10^{3}$, adiabatic bottom, 2D simulations): a) plot of signals provided by numerical probes, b) snapshot of streamlines and c) temperature field at $\mathrm{t}=3$.

First, the disturbance with smaller frequency disappears, leaving the two "external” regions (preand post-obstruction) free of oscillations. This can be seen for $\mathrm{Ra}=3000$ in Fig. 7. In such conditions, only the large-frequency disturbance survives (it is clearly visible for $A_{X} / 4<x<3 A_{X} / 4$ ).

A further increase in the Rayleigh number, however, causes the suppression of this high-frequency disturbance as well (Fig. 8, Ra=5000), making the flow completely steady.

Replacement of the adiabatic bottom wall with a conducting bottom has a non-negligible impact on such scenario. The frequency spectrum for $\mathrm{Ra}=1000$, indeed, is even more complex. It includes three clearly recognizable frequencies (Fig. 9). 


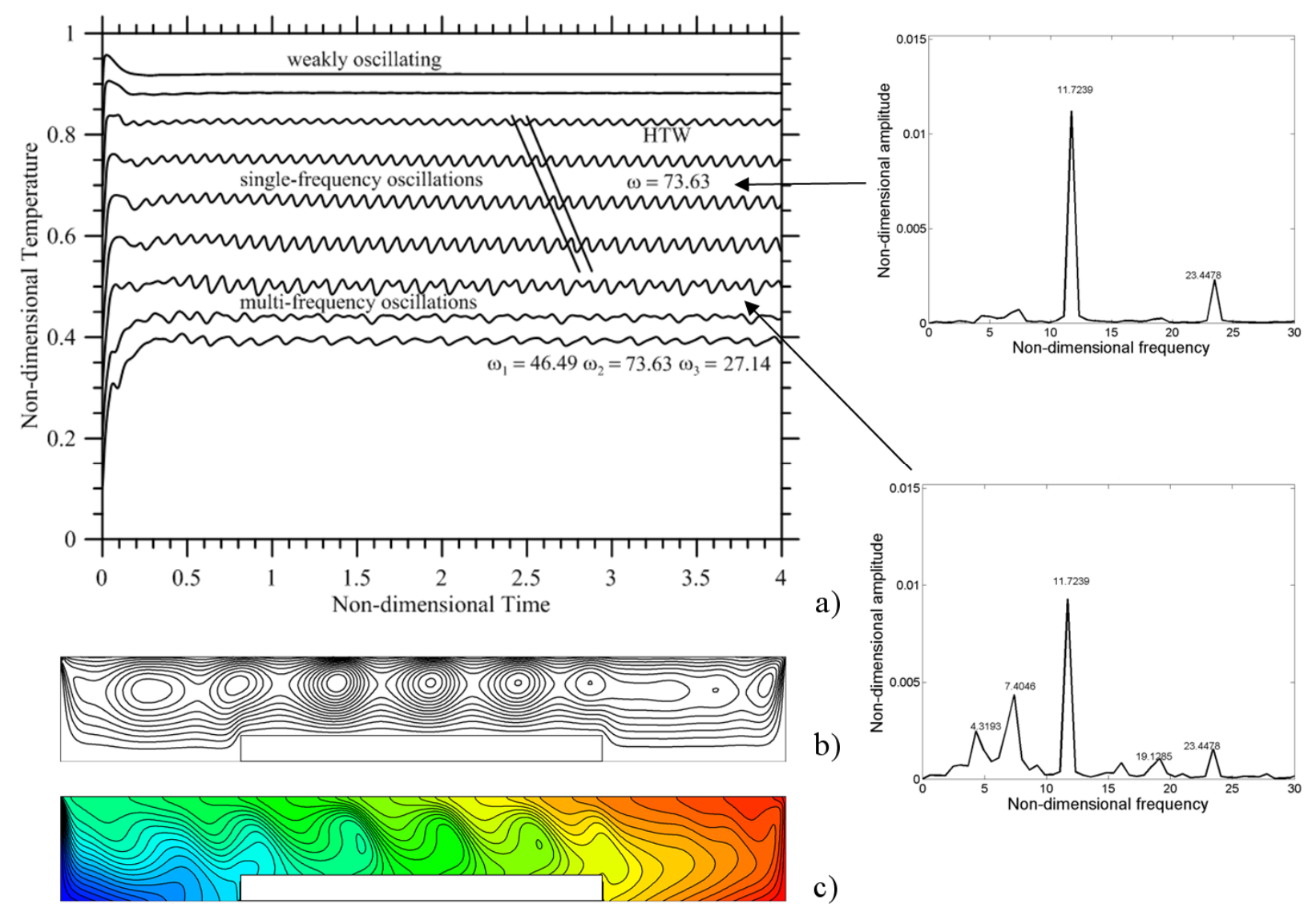

Figure 9: $\left(\operatorname{Pr}=15, A_{x}=20\right.$, central obstruction, $\Gamma_{1}=0.25, \Lambda=0.25, M a=2.0 \times 10^{4}, \quad R a=1 \times 10^{3}$, conducting bottom, 2D simulations): a) plot of signals provided by numerical probes with related frequency spectra, b) snapshot of streamlines and c) temperature field at $t=3$.

In this case the evolutionary progress towards the steady state seems to be articulated into three distinct stages. Moreover, the system experiences a significant increase in the threshold to be exceeded (in terms of value of the Rayleigh number) to enable the back transition to steady state. This is illustrated in Figs. 10-11. 


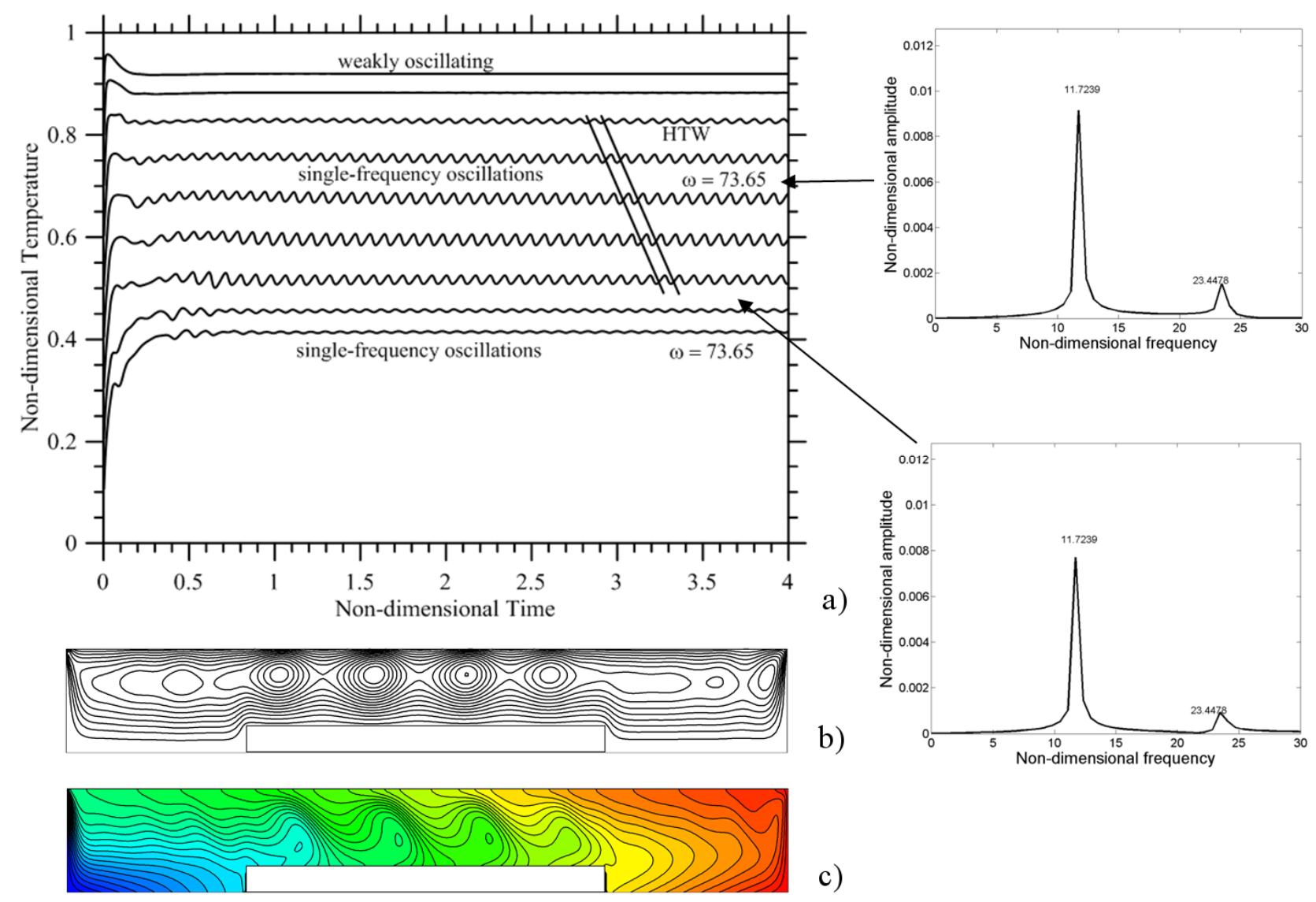

Figure 10: $\left(\operatorname{Pr}=15, \mathrm{~A}_{\mathrm{x}}=20\right.$, central obstruction, $\Gamma_{1}=0.25, \Lambda=0.25, \mathrm{Ma}=2.0 \times 10^{4}, \mathrm{Ra}=3 \times 10^{3}$, conducting bottom, 2D simulations): a) plot of signals provided by numerical probes with related frequency spectra, b) snapshot of streamlines and c) temperature field at $t=3$.

Initially, for $\mathrm{Ra}=3000$ (Fig. 10), the frequency spectrum with three frequencies $\left(\omega_{1}=46.49\right.$, $\omega_{2}=73.63$ and $\omega_{3}=27.14$ obtained for $\left.\mathrm{Ra}=1000\right)$ is taken over by a two-frequency state in which $\omega_{3}$ is no longer present. A further increase in Ra makes $\omega_{2}$ the only surviving frequency $\left(\omega_{2}=74.79\right.$ for $\mathrm{Ra}=5000$, not shown, and $\omega_{2}=77.53$ for $\mathrm{Ra}=7000$ see Fig. 11 ). The amplitude of the temperature signals behaves as a decreasing function of Ra until a completely steady state is attained for $\mathrm{Ra} \cong 7800$. 


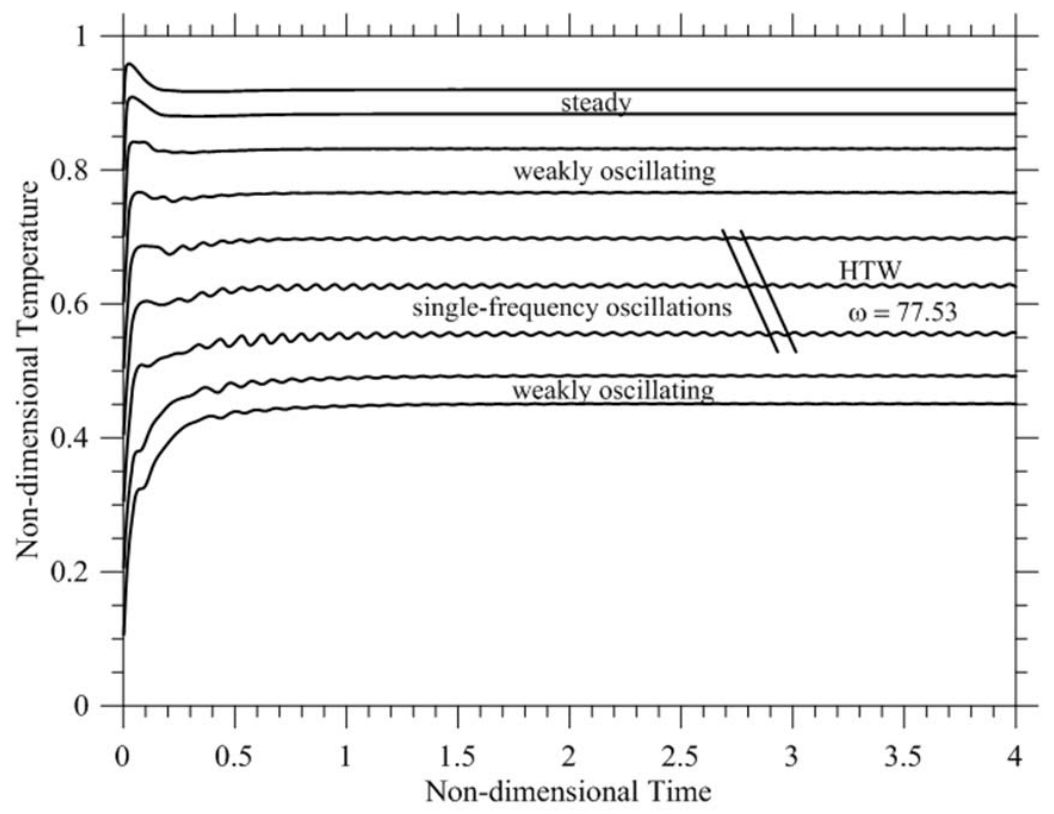

a)

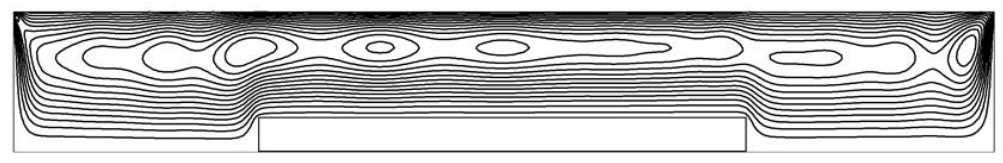

b)

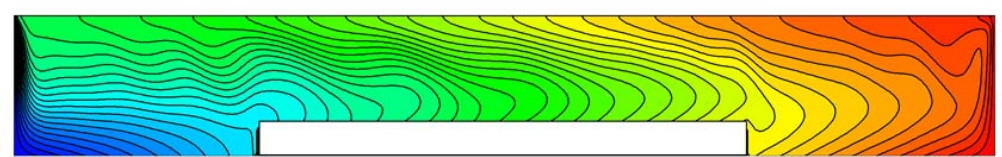

c)

Figure 11: $\left(\operatorname{Pr}=15, A_{x}=20\right.$, central obstruction, $\Gamma_{1}=0.25, \Lambda=0.25, M a=2.0 \times 10^{4}, \quad R a=7 \times 10^{3}$, conducting bottom, 2D simulations): a) plot of signals provided by numerical probes, b) snapshot of streamlines and c) temperature field at $\mathrm{t}=3$.
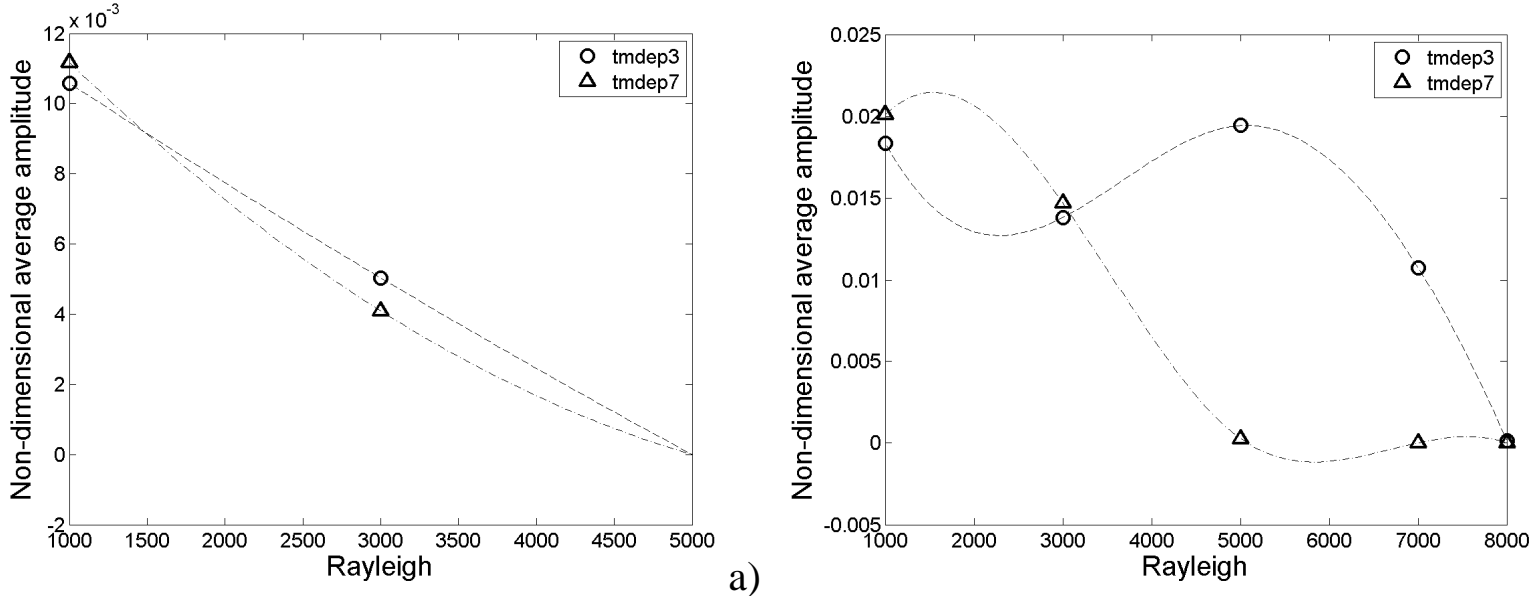

b)

Figure 12: Non-dimensional temperature signal amplitude as a function of the Rayleigh number for the backward-facing step case $(\Lambda=0.25)$ : a) adiabatic bottom, b) conducting bottom. 

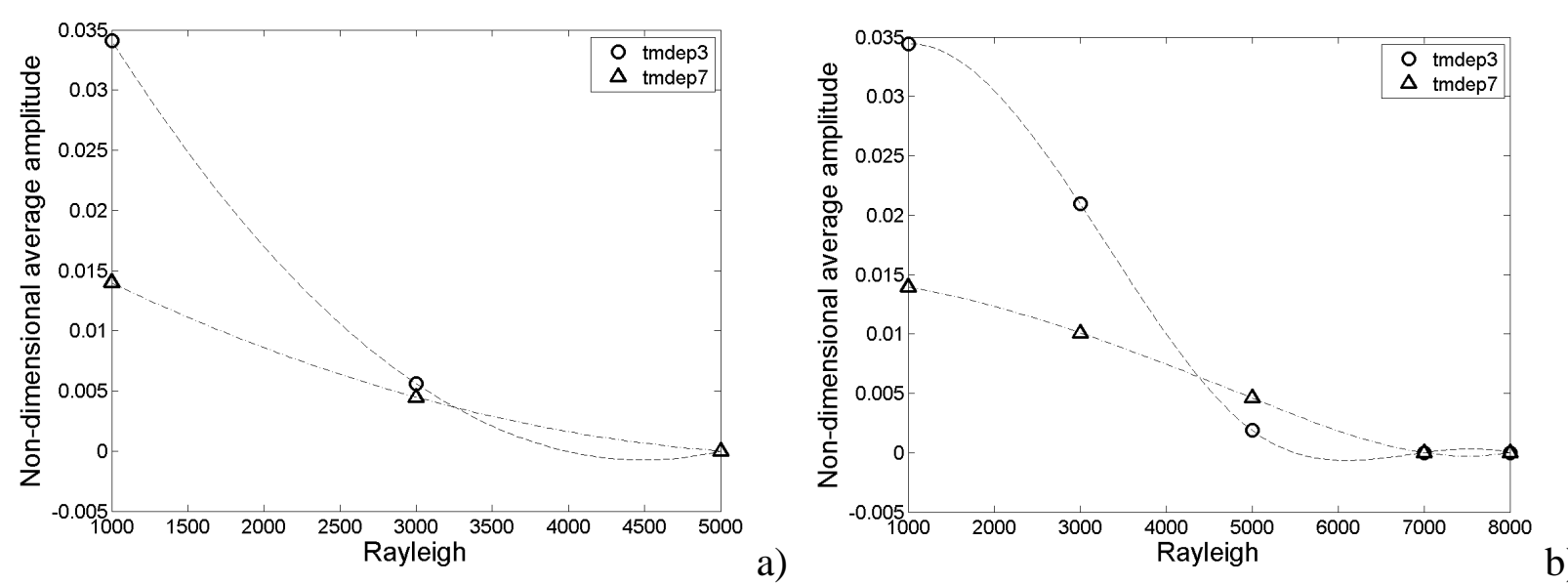

Figure 13: Non-dimensional temperature signal amplitude as a function of the Rayleigh number for the forward-facing step case $(\Lambda=0.25)$ : a) adiabatic bottom, b) conducting bottom.
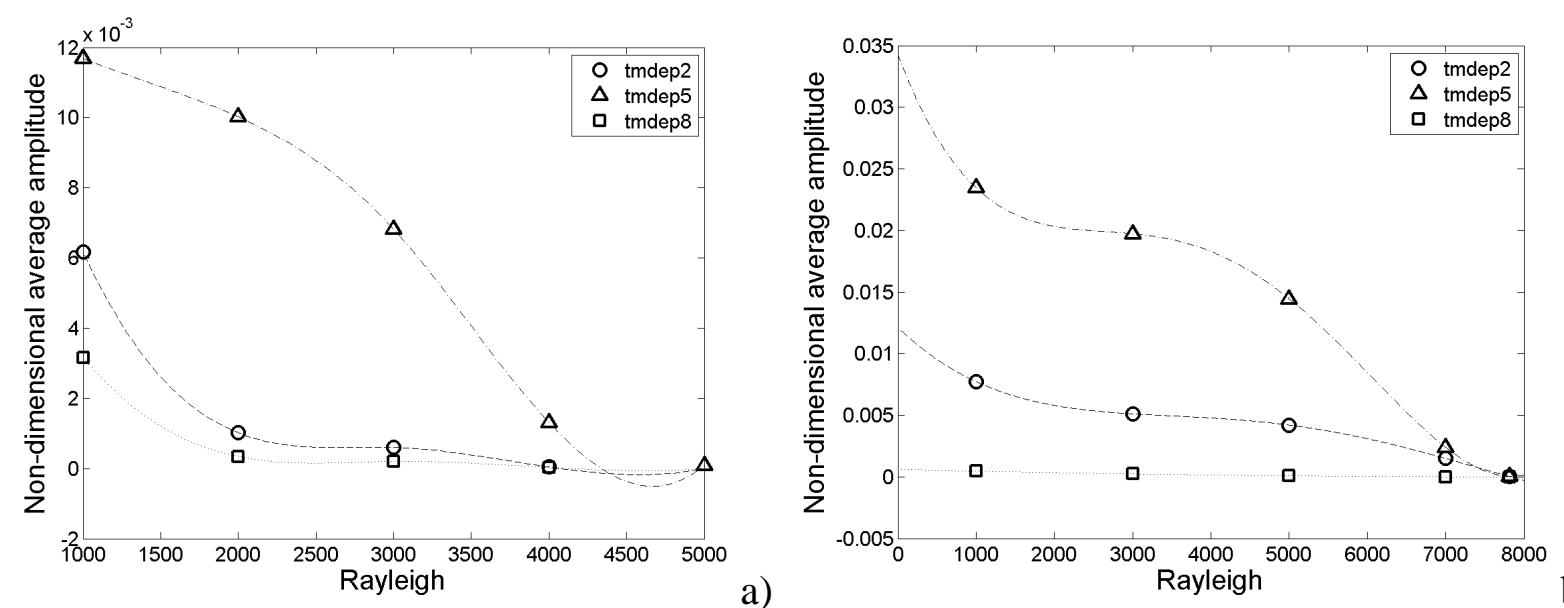

Figure 14: Non-dimensional temperature signal amplitude as a function of the Rayleigh number for the case with central obstruction $(\Lambda=0.25)$ : a) adiabatic bottom, b) conducting bottom.

A change in the location of the step does not influence such trends significantly from a qualitative point of view (the reader being referred to Figs. 12-14 for related quantitative details). The threshold (in terms of Rayleigh number) to be exceeded to induced back transition to a steady state is always higher $(60 \%)$ in the case with conducting wall ( $\mathrm{Ra} \cong 5 \times 10^{3}$ for adiabatic bottom and $\mathrm{Ra} \cong 8 \times 10^{3}$ for conducting bottom).

\subsection{The influence of thermal and geometrical conditions}

An increase in the height of the step has generally a damping influence on the flow, accelerating dramatically the progression towards stationary conditions. For $\Lambda=0.45$ the flow above the step is always steady or weakly oscillating. Such information can be gathered from Tables II, III and IV, which summarize the results for the backward-facing, forward-facing and central obstruction cases, respectively, for different thermal and geometrical conditions. 


\begin{tabular}{ccccc}
\hline \hline Ra & Bottom wall BC & $\Lambda$ & $\omega_{\mathrm{T} 3}(\cong)$ & $\omega_{\mathrm{T} 7}(\cong)$ \\
\hline \hline $1 \times 10^{3}$ & Adiabatic & 0.25 & 72.8 & 46.8 \\
& Conducting & 0.25 & $27-46.5-73.6$ & 46.5 \\
& Adiabatic & 0.45 & 0 & 46.8 \\
& Conducting & 0.45 & 0 & 46.5 \\
$3 \times 10^{3}$ & Adiabatic & 0.25 & 77.5 & 46.5 \\
& Conducting & 0.25 & $27-46.5-73.6$ & 46.5 \\
& Adiabatic & 0.45 & 0 & 0 \\
& Conducting & 0.45 & 0 & 46.5 \\
$5 \times 10^{3}$ & Adiabatic & 0.25 & 0 & 0 \\
& Conducting & 0.25 & 73.6 & 46.5 \\
& Adiabatic & 0.45 & 0 & 0 \\
& Conducting & 0.45 & 0 & 0 \\
$6 \times 10^{3}$ & Adiabatic & 0.25 & 0 & 0 \\
& Conducting & 0.25 & 73.6 & $<<1$ \\
& Adiabatic & 0.45 & 0 & 0 \\
& Conducting & 0.45 & 0 & 0 \\
$7 \times 10^{3}$ & Adiabatic & 0.25 & 0 & 0 \\
& Conducting & 0.25 & 73.6 & 0 \\
& Adiabatic & 0.45 & 0 & 0 \\
& Conducting & 0.45 & 0 & 0 \\
\hline \hline
\end{tabular}

Table II: Summary of numerical results (2D simulations) for the case with the backward-facing step $\left(\operatorname{Pr}=15, A_{x}=20, M a=20000\right)$

\begin{tabular}{ccccc}
\hline \hline $\mathrm{Ra}$ & Bottom wall BC & $\Lambda$ & $\omega_{\mathrm{T} 3}(\cong)$ & $\omega_{\mathrm{T} 7}(\cong)$ \\
\hline \hline $1 \times 10^{3}$ & Adiabatic & 0.25 & 43.2 & 70.3 \\
& Conducting & 0.25 & $27-43.2-70.3$ & 70.3 \\
& Adiabatic & 0.45 & 46.5 & $<<1$ \\
& Conducting & 0.45 & 46.5 & 0 \\
$3 \times 10^{3}$ & Adiabatic & 0.25 & 48.6 & 75.7 \\
& Conducting & 0.25 & $27.1-42.6-73.6$ & 70.3 \\
& Adiabatic & 0.45 & 0 & 0 \\
& Conducting & 0.45 & 46.5 & 0 \\
$5 \times 10^{3}$ & Adiabatic & 0.25 & 0 & 0 \\
& Conducting & 0.25 & $46.5-70.3$ & 70.3 \\
& Adiabatic & 0.45 & 0 & 0 \\
& Conducting & 0.45 & 0 & 0 \\
\hline \hline
\end{tabular}

Table III: Summary of numerical results (2D simulations) for the case with the forward-facing step $\left(\mathrm{Pr}=15, \mathrm{~A}_{\mathrm{x}}=20, \mathrm{Ma}=20000\right)$ 


\begin{tabular}{cccccc}
\hline \hline Ra & Bottom wall BC & $\Lambda$ & $\omega_{\mathrm{T} 2}(\cong)$ & $\omega_{\mathrm{T} 5}(\cong)$ & $\omega_{\mathrm{T} 8}(\cong)$ \\
\hline \hline $1 \times 10^{3}$ & Adiabatic & 0.25 & 45.2 & 76.3 & $<<1$ \\
& Conducting & 0.25 & $27.1-46.5-73.6$ & 73.6 & $<<1$ \\
& Adiabatic & 0.45 & 0 & 0 & 0 \\
$3 \times 10^{3}$ & Conducting & 0.45 & $<<1$ & 0 & 0 \\
& Adiabatic & 0.25 & $<<1$ & 76.1 & $<<1$ \\
& Conducting & 0.25 & 73.6 & 73.6 & $<<1$ \\
& Adiabatic & 0.45 & 0 & 0 & 0 \\
$5 \times 10^{3}$ & Conducting & 0.45 & 0 & 0 & 0 \\
& Adiabatic & 0.25 & 0 & 0 & 0 \\
& Conducting & 0.25 & 74.8 & 74.8 & $<<1$ \\
& Adiabatic & 0.45 & 0 & 0 & 0 \\
$7 \times 10^{3}$ & Conducting & 0.45 & 0 & 0 & 0 \\
& Adiabatic & 0.25 & 0 & 0 & 0 \\
& Conducting & 0.25 & $<<1$ & 77.5 & $<<1$ \\
& Adiabatic & 0.45 & 0 & 0 & 0 \\
& Conducting & 0.45 & 0 & 0 & 0 \\
\hline \hline
\end{tabular}

Table IV: Summary of numerical results (2D simulations) for the case with the central obstruction $\left(\operatorname{Pr}=15, A_{x}=20, M a=20000\right)$

Notably, sifting through such tables and using Figs. 12-14, some general features about the frequency and amplitude variations of the temperature signals can be identified, as further discussed in the following:

\section{Adiabatic bottom}

For the backward configuration, the frequency spectrum displayed by the temperature signals becomes simpler and the frequency decreases as soon as the HTW gets off the step; for small Ra the signals amplitude increases slightly or stays almost constant after the step, while for large Ra it tends to decrease. For the forward-facing step case, the frequency increases and the amplitude decreases above the step. On the mid-cross section, the frequency spectrum becomes more complex, with the presence of more than one frequency. The obstruction case presents a different trend, with a visible increase of both frequency and amplitude above the obstruction. When the Rayleigh number is increased, it affects essentially the signals in terms of amplitudes (which decrease until the steady state is reached).

\section{Conducting bottom}

The conducting boundary condition produces an increase in the amplitude and the number of the travelling rolls, i.e. the wave number; it also induces a broadening of the frequency spectrum close to the cold side (the values cover the range from $\omega_{\mathrm{HTW}} \cong 27$ to $\omega_{\mathrm{HTW}} \cong 75$ for small $\mathrm{Ra}$ ). On the contrary, it tends to make the flow more stable in proximity to the hot side. This apparently counterintuitive behavior can be explained by examining the temperature distribution along the cavity; 
indeed, it is possible to recognize near the cold side an almost horizontal temperature stratification with a vertical temperature gradient that is opposite to gravity (therefore there is less buoyancy convection, which can explain why the HTW can still exist in that region at relatively high values of the Rayleigh number; vice versa the vertical component of the temperature gradient has opposite sense close to the hot side). In general, the frequency spectrum is relatively complex at lower Ra, but as soon as the Rayleigh number is increased, it becomes simpler, with only one frequency. Then the amplitude decreases until the steady state is attained. The value of Ra required to recover the steady state, however, is much larger than that needed for the adiabatic-bottom case.

The following general conclusions can therefore be drawn for the considered dynamics:

The frequency spectrum is more complex in the conducting boundary case, especially in proximity to the cold wall, due to the local weakening of buoyancy flow caused by the aforementioned horizontal temperature stratification (this can be noticed, for instance, in Fig. 2, where the temperature fields and the streamlines of both the adiabatic and the conducting conditions are compared). As shown in Fig. 12, Fig. 13 and Fig. 14 the amplitude of the HTW is larger in the conducting case.

Moreover:

An increase in the Rayleigh number has a two-fold effect: In first place, in agreement with existing experiments, for both adiabatic and conducting boundary conditions at the bottom, it leads to the suppression of the HTW. As witnessed by Figs. 12-14, the non-dimensional average amplitude of the temperature oscillations decreases as a function of the Rayleigh number and the oscillations are completely damped when a given threshold is reached ( $\mathrm{Ra} \cong 5 \times 10^{3}$ adiabatic bottom case, $\mathrm{Ra} \cong 8 \times 10^{3}$ conducting bottom case); secondly, for the conducting bottom condition, as the Rayleigh number grows it leads to a simplification of the frequency spectrum. As evident in Tables II, III and IV, at low Ra multiple angular frequencies can be measured in proximity of the cold wall, whereas only one frequency prevails at higher Ra, until the amplitude of oscillations becomes zero.

The following specific behaviors also deserve attention:

- For the obstruction case with $\Lambda=0.25$ and conducting bottom, the HTW exists essentially above the step and the flow is steady or weakly oscillatory elsewhere. This can be seen in the diagrams of the temperature signals shown in Figs. 9-11, where it is possible to recognize above the step the typical continuous phase-shift of the temperature peaks, which denotes the presence of the HTW. This effect also occurs in the adiabatic case, provided the Rayleigh number is not too high $\left(\mathrm{Ra}=10^{3}\right.$ and $\mathrm{Ra}=3 \times 10^{3}$ in Fig. 6 and Fig. 7, respectively);

- The obstruction case for $\Lambda=0.45$ shows for both the boundary conditions steady flow regardless of the considered value of the Rayleigh number (except for the conducting case at $\mathrm{Ra}=10^{3}$ for which there are weakly oscillating rolls before the obstruction, but no HTW).

Therefore, the following additional meaningful conclusions can be elaborated:

- The reason for which the wave appears only above the obstacle relates to the damping influence that buoyancy exerts on it. Due to the reduced extension (in the vertical direction) 
of the fluid layer above the obstacle, the wave can survive in that region whereas it is annihilated where the fluid depth is larger (this argument is confirmed by the relatively small values of the Rayleigh number for which the wave is suppressed in the absence of the blockage, this being $\cong 1500$ and 5500 for adiabatic and conducting bottom walls, respectively).

- An increase in the thickness of the obstruction, however, can also have a stabilizing effect. In other words, for a relatively thick obstruction, an increase in its vertical extension (at fixed $\mathrm{Ra}$ ) is equivalent to an increase in the Rayleigh number (for a fixed thickness). At a certain stage, both can lead to suppression of the HTW.

As a concluding remark for this section we also wish to highlight that, according to the present results, an interpretation of the dynamics displayed by the flow in different regions (with different cross-sectional area) pretending that they are uncoupled (i.e. trying to apply locally earlier results available for unobstructed geometries such as that by Parmentier et al. [11]) is not straightforward as one would imagine. All our attempts in this regard were unsuccessful. This can be justified taking into account the aforementioned intrinsic nature of Marangoni flow and the HTWs for highPr numbers. Due to the dominant component of the HTW directed in the upstream direction, trying to interpret the dynamics pretending that some areas are disjoint and do not influence each other is not possible.

\subsection{Stationary Waves}

Having completed a description of the hierarchy of spatio-temporal behaviors for increasing values of the Rayleigh number, and related sensitivity to thermal and geometrical factors, we turn now to an assessment (or verification) of the effective properties of the flow in the cavity when it is allowed to be $3 \mathrm{D}$ and the same conditions for which 2D simulations give steady flow are considered.

As shown in Figs. 15-18 for the same values of the longitudinal aspect ratio $\mathrm{A}_{\mathrm{z}}$ assumed previously $\left(A_{z}=15\right)$ and distinct geometrical configurations, the states with steady flow predicted by 2D computations are retained when the constraint of two-dimensionality is removed.

In the following, we refer to the spanwise velocity (w) since the effect produced by the finite extension of the domain along the $\mathrm{z}$ axis can be seen especially in the iso-surfaces of this velocity component. As evident in the figures, the highest value of $\mathrm{w}$ can be found near the walls at $\mathrm{z}=0$ and $\mathrm{z}=\mathrm{A}_{\mathrm{z}}$, which indicates that $3 \mathrm{D}$ effects are relatively limited and localized essentially in proximity to these solid boundaries.

Interesting insights can also be gathered from the iso-surfaces of the vertical component of velocity (v). For all cases such isosurfaces give evidence for what may be regarded as a "stationary wave".

At this stage we wish to emphasize that this specific flow configuration for which the flow displays alternating maxima and minima along the horizontal direction, which are fixed in time (obtained 
here in the framework of direct numerical solution of the governing equations in their complete, non-linear and time-dependent form), is in agreement with the predictions of dedicated stability analyses based on the concept of "absolute stability” (Priede and Gerbeth [50]; Pérez-García et al. [51]).

Notably, the principal idea at the root of these theoretical studies is that "distant lateral boundaries may be regarded as a permanent stationary disturbance of the homogeneous basic state”.
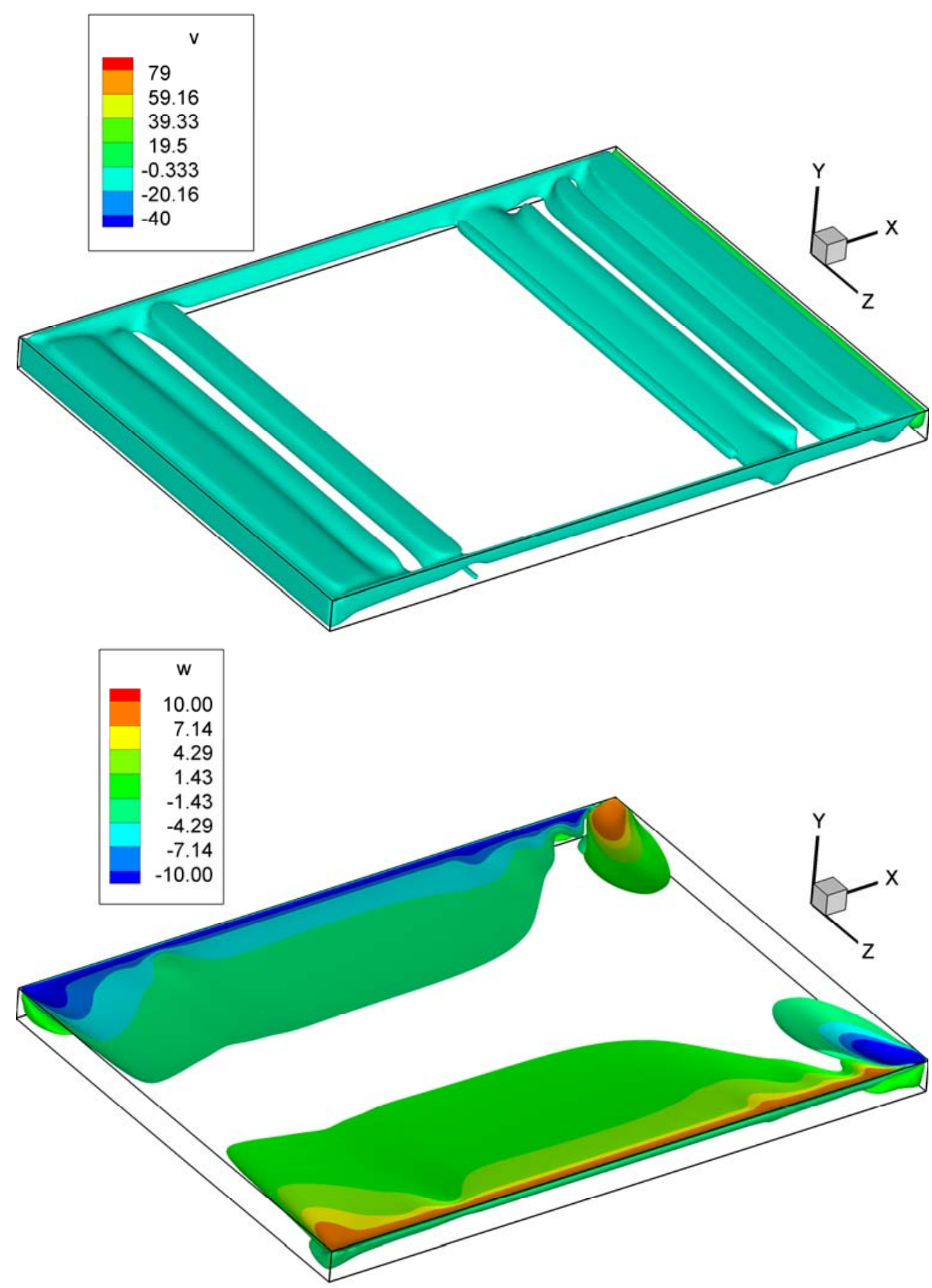

a)

b)

Figure 15: Obstruction in the center, $\Lambda=0.25$, adiabatic bottom wall ( $\mathrm{Ma}=20000, \mathrm{Ra}=8000, \mathrm{~A}_{\mathrm{z}}=15$, $\mathrm{t}=5$ ): a) velocity component along y (v component), b) velocity component along $\mathrm{z}$ (w component). The numerical results reveal that though some departure from two-dimensionality occurs, such effects are concentrated in proximity to the wall along the entire depth of the cavity 


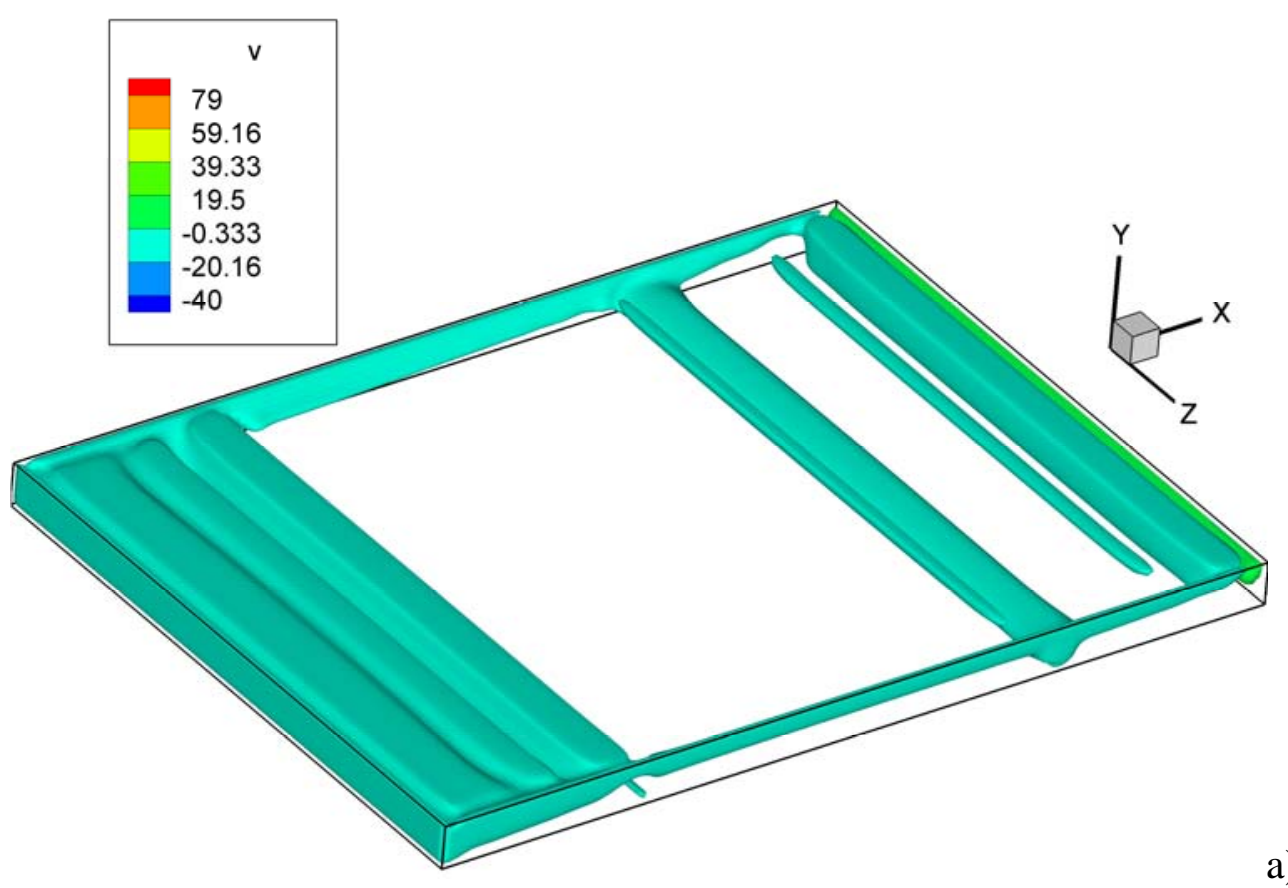

a)

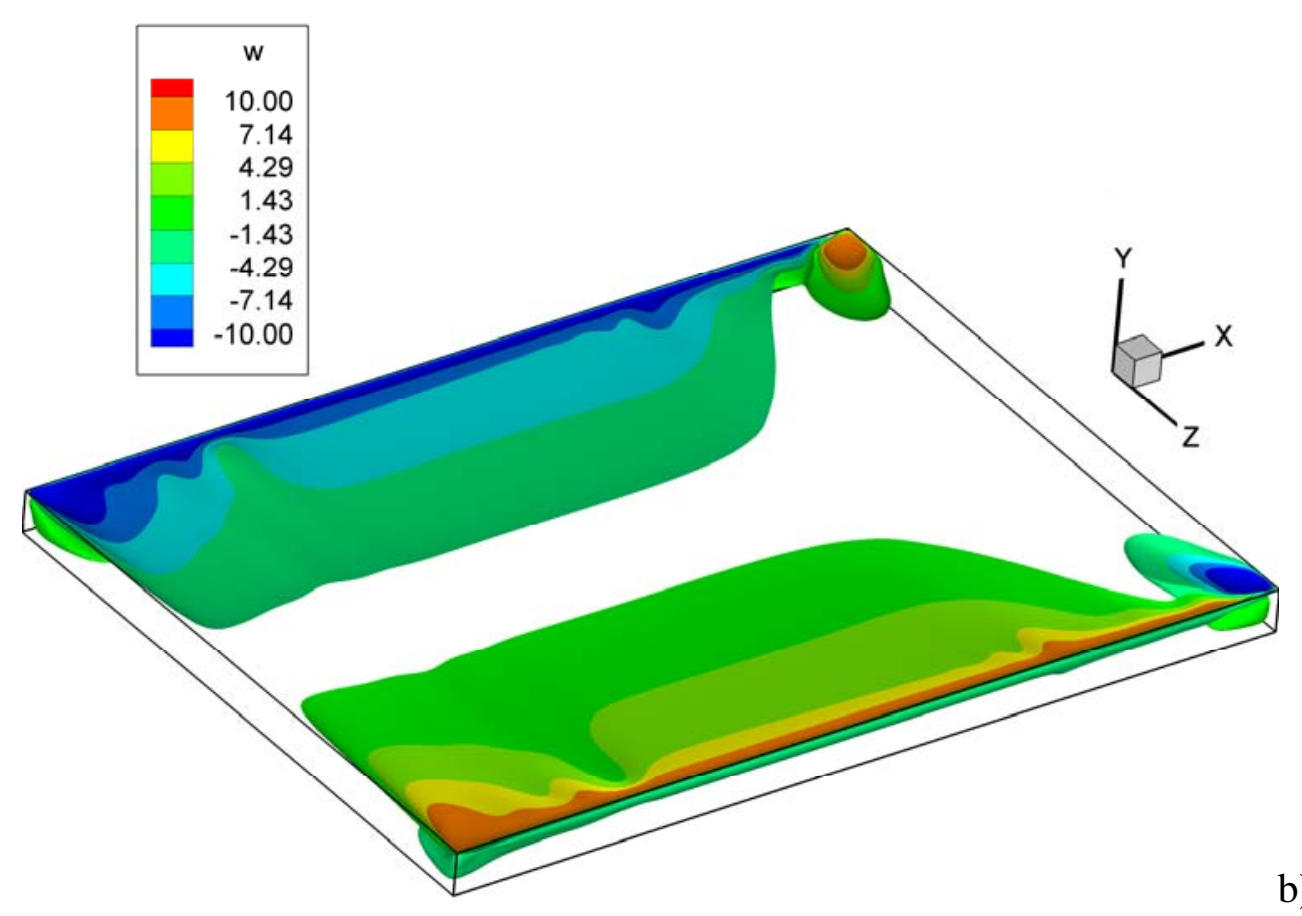

b)

Figure 16: Obstruction in the center, $\Lambda=0.25$, conducting bottom wall (Ma=20000, $\mathrm{Ra}=8000, \mathrm{~A}_{\mathrm{z}}$ $=15, \mathrm{t}=5)$ : a) velocity component along y ( $\mathrm{v}$ component), b) velocity component along $\mathrm{z}(\mathrm{w}$ component).

The ability of lateral walls to disturb the assumed uniformity of the basic flow was initially assessed by Priede and Gerbeth [50]. These authors also evaluated how far this perturbation can spread from a vertical boundary, revealing that a sidewall can cause a stationary wave pattern when a zerofrequency mode is excited. 
The difference between this specific mode of convection and the other possible stationary modes predicted for $\operatorname{Pr}>1$ in the earlier linear stability analyses by Gershuni et al. [10] and Mercier and Normand [12] lies in the fact that while in those studies the disturbances were found to be essentially longitudinal (rolls with axes parallel to the imposed temperature gradient) and to emerge solely for non-adiabatic free surface (modelled as a conducting boundary in the study of Gershuni et al. [10]; characterized by a given value of the Biot number in the analysis by Mercier and Normand [12]), the stationary patterns seen in the present study are essentially transverse ( $\Phi=09$ and, as predicted by Priede and Gerbeth [50], they can occur even in the limit $\mathrm{Bi}=0$. For pure Marangoni flow with adiabatic horizontal boundaries, these authors found such modes to become possible and spread over the whole layer regardless of its extent for $\operatorname{Pr}>0.67$ (for a sufficiently high value of the Marangoni number). In particular, for $\operatorname{Pr}=13.9$ and under the effect of buoyancy the stationary perturbation due to the upstream end wall was found to become unstable and spread over the whole layer before the onset of oscillatory convection for $\mathrm{Bo}_{\mathrm{dyn}}=\mathrm{W}^{-1}>0.2$. Similarly, for the same value of the Prandtl number and $A_{x} \cong 25$, by 2D simulations Shevtsova et al. [6] observed steady multicellular states to replace the HTW for $\mathrm{Bo}_{\mathrm{dyn}}=\mathrm{W}^{-1}>0.3$, which is also in agreement with the present simulations (for which we assumed $\mathrm{Bo}_{\mathrm{dyn}}=\mathrm{W}^{-1}=0.4$ ).

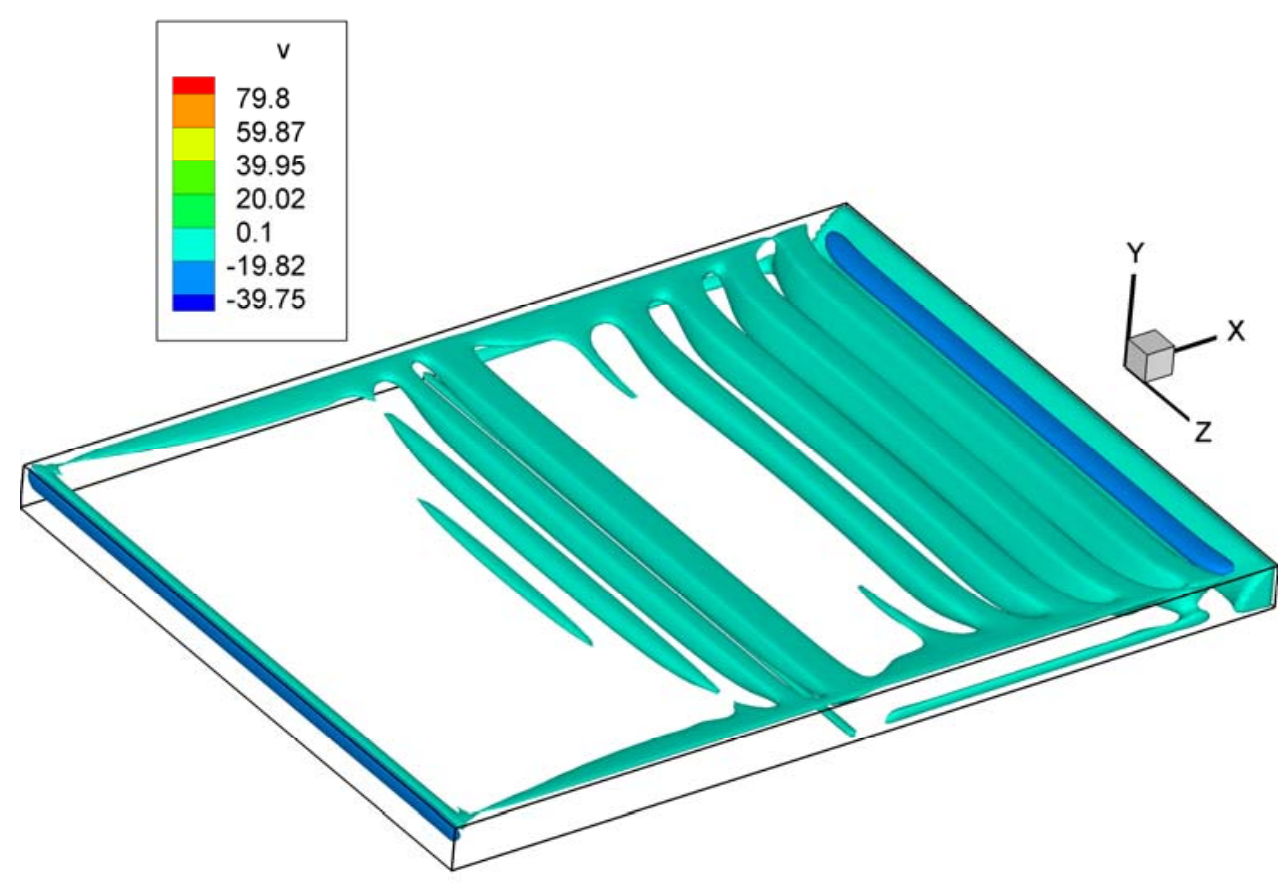

Figure 17: Backward Facing step, $\Lambda=0.25$ : velocity component along y (v component) for adiabatic bottom wall (Ma=20000, $\left.\mathrm{Ra}=8000, \mathrm{~A}_{\mathrm{z}}=15, \mathrm{t}=5\right)$. 


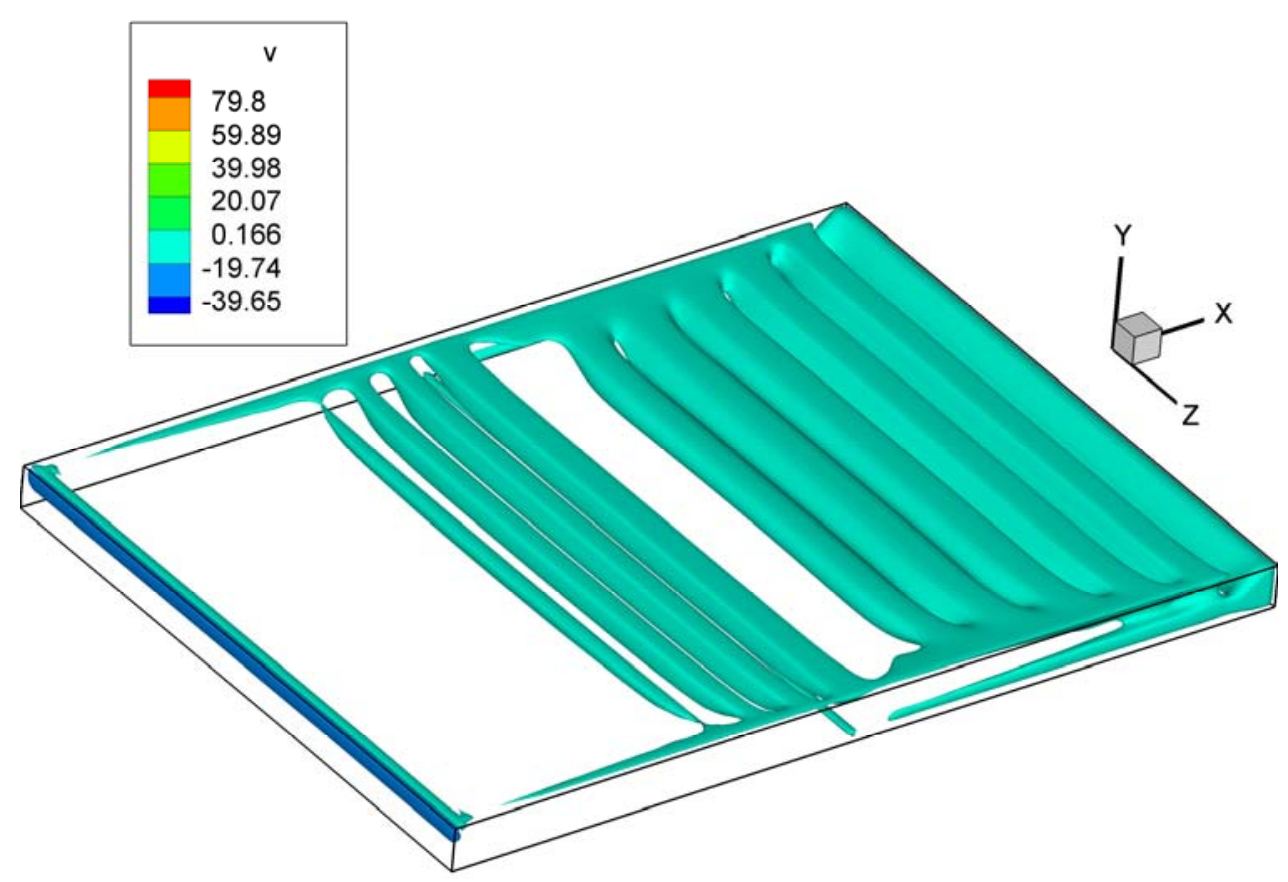

Figure 18: Backward Facing step, $\Lambda=0.25$ : velocity component along y ( $\mathrm{v}$ component) for conducting bottom wall ( $\mathrm{Ma}=20000, \mathrm{Ra}=8000, \mathrm{~A}_{\mathrm{z}}=15, \mathrm{t}=5$ ).

As a 'new aspect' with respect to earlier studies, according to the present numerical results, such stationary waves need not to originate necessarily from walls that physically prevent the flow from developing along a given direction. Remarkably, they can also be produced by a sudden variation in the available cross section, as witnessed by the presence of a second set of disturbance nodes (in addition to that spatially spreading from the hot wall in the downstream direction) originating from the station where the geometry variation occurs (Figs. 15-18). These two regions are separated by a disturbance-free region. The disturbance nodes at different stations in the spanwise direction align almost perfectly along the $\mathrm{z}$ direction thereby confirming that the stationary wave is transverse. We could detect no signature of the HTWs like those shown in Figs. 3c and 4c on the free surface for such conditions.

\subsection{The assumption of undeformable liquid-gas interface and its validity}

All the results presented in our work have been obtained under quite a severe approximation, i.e. the assumption of perfectly horizontal and undeformable liquid-gas interface.

At this stage meaningful exploitation of such findings would require a proper assessment of the conditions for which such a simplification can be assumed to hold.

These conditions can be derived on the basis of the typical results of the order of magnitude analysis (OMA) or similar strategies. 
The OMA is typically based on a comparative examination of the relative importance or strength of the forces acting on the interface. These are: gravity, surface tension and viscous forces due to the fluid motion itself produced by the other two forces.

Surface tension is responsible for the generation of Marangoni flow. A similar influence can be ascribed to gravity. In the presence of a horizontal temperature gradient it is responsible for the onset of buoyancy flow while at the same time it tends to constrain the liquid to fill the cavity and to form a top planar interface separating it from the less dense upper fluid (air).

The relative importance of all these effects can be measured by means of relevant non-dimensional (characteristic) numbers. More specifically, the parameter accounting for the relative strength of viscous forces (due to thermal convection), which tend to deform dynamically the free interface and surface tension is the so-called Capillary number:

$$
C a=\frac{\mu V_{r}}{\sigma}
$$

where $\sigma$ is the liquid surface tension and $V_{r}$ is a proper reference velocity and $\mathrm{d}$ has to be considered as the distance between the free surface and the bottom wall. Similarly, the convective Galileo number accounts for the relative strength of viscous forces and gravity

$$
G a_{c}=\frac{\mu V_{r}}{\Delta \rho g d^{2}}
$$

where $\Delta \rho$ is the density difference between the considered liquid and air ( $\Delta \rho \cong \rho$ where $\rho$ is the silicone oil density, shown in Table I).

On the basis of the OMA (see, e.g., Burguete et al. [25]; Golovin et al. [52] and Borcia and Bestehorn [53]) it can be inferred that when $C a<1$ or $G a_{c}<1$, the non-dimensional deformation $\delta$ experienced by a free surface has to be of the order of $\delta=\mathrm{O}(C a)$ or $\delta=\mathrm{O}\left(G a_{c}\right)$, respectively. In the problem considered here, both gravity and surface tension act as forces counteracting the dynamic deformation of the free surface, which implies a OMA "hierarchy", i.e. the problem may be divided in two parts, one being devoted to situation in which surface tension becomes the prevailing force or the case of gravity-dominated dynamics. The relative strength of these two effects depends on the so-called Bond number, generally defined in the literature as:

$$
B o=\frac{\Delta \rho g d^{2}}{\sigma} \cong \frac{\rho g d^{2}}{\sigma}
$$

Gravity and surface tension are equivalent when $B o=1$, which for the present conditions (see again Table I) would be corresponding to a layer having a depth of $\cong 0.15 \mathrm{~cm}$.

First, we address the case $B o<1$ (dominant surface tension), i.e. layers with a relatively small depth $(<0.15 \mathrm{~cm})$. 
In the case of surface-tension-driven flow, the reference velocity $V_{r}$ must be obviously assumed to be the so-called Marangoni velocity

$V_{M a}=\frac{\sigma_{T} \Delta T}{\mu}$

The relevant parameter to be considered in this regime, namely the Capillary number based on the characteristic Marangoni velocity, therefore reads

$C a_{M a}=\frac{\sigma_{T} \Delta T}{\sigma}$

Because thermal buoyancy is also present, an equivalent definition can be introduced by using the so-called buoyancy velocity

$V_{g}=\frac{g \beta_{T} \Delta T d^{2}}{v}$

The equivalent Capillary number, therefore, reads

$C a_{R a}=\frac{\rho g \beta_{T} \Delta T d^{2}}{\sigma}=\frac{B o R a}{\operatorname{Pr} G a}$

where $G a$ is the classical Galileo number $\left(\mathrm{gd}^{3} / \mathrm{v}^{2}\right)$.

In the other limiting condition, in which gravity becomes the most significant restoring force (d> $0.15 \mathrm{~cm}$ ), the relevant convective Galileo number, similarly, will read:

$G a_{M a}=\frac{M a}{\operatorname{Pr} G a}$

or

$G a_{R a}=\frac{R a}{\operatorname{Pr} G a}$

according to whether it is based on the characteristic Marangoni or buoyancy velocity, respectively. The values taken by both the Capillary number and the convective Galileo number for increasing values of the depth d can be found in Table V: 


\begin{tabular}{lllllll}
\hline \hline depth $[\mathrm{cm}]$ & $B o$ & $R a$ & $C a_{M a} \times 10$ & $C a_{R a} \times 10^{2}$ & $G a_{M a}$ & $G a_{R a} \times 10$ \\
\hline \hline 0.01 & $5 \times 10^{-3}$ & 36.2 & 6.10 & 0.11 & 136 & 2.460 \\
0.05 & 0.12 & 905 & 1.22 & 0.57 & 1.088 & 0.492 \\
0.08 & 0.26 & 2036 & 0.813 & 0.85 & 0.322 & 0.328 \\
0.10 & 0.46 & 3620 & 0.610 & 1.14 & 0.136 & 0.246 \\
0.13 & 0.72 & 5656 & 0.488 & 1.42 & 0.0696 & 0.197 \\
0.15 & 1.04 & 8145 & 0.407 & 1.71 & 0.0403 & 0.164 \\
0.17 & 1.42 & 11086 & 0.348 & 1.99 & 0.0254 & 0.141 \\
0.20 & 1.85 & 14480 & 0.305 & 2.27 & 0.0170 & 0.123 \\
\hline \hline
\end{tabular}

Table V: Bond number, Rayleigh number, Capillary number and convective Galileo number as a function of the layer depth for $\mathrm{Ma}=20000$.

Therefore, one may argue that for the considered fluid (silicone oil) the interface can be assumed flat and horizontal over a wide range of conditions (in practice, as shown in Table V, a lower boundary to the applicability of the present results is represented by the constraint that, in order to prevent the Capillary number from exceeding values $\mathrm{O}\left(10^{-1}\right)$, the minimum distance between the free surface and the bottom wall should not be smaller than $0.05 \mathrm{~cm}$ (for this value of $\mathrm{d}, \mathrm{Bo}=\mathrm{O}\left(10^{-}\right.$ $\left.{ }^{1}\right), R a=\mathrm{O}\left(10^{3}\right)$ and $\left.C a=\mathrm{O}\left(10^{-1}\right)\right)$.

An additional check, however, is required for the case in which buoyancy convection becomes significant. The "worst conditions" in our case (see Sect. 3) correspond to $R a=8000$. As shown in table $\mathrm{V}$, this would be equivalent to considering a layer with depth $\cong 0.15 \mathrm{~cm}$ for which $B o \cong 1$. Table V yet confirms that all parameters relevant to this case $\left(C a_{M a}, C a_{R a}, G a_{M a}, G a_{R a}\right)$ would be $<<1$ for the conditions considered in the present study.

\section{Conclusions}

Mixed (hybrid) thermocapillary-thermogravitational convection is a canonical subject of investigation in nonlinear dynamics and the variety of associated fluid-dynamic behaviors are often considered as archetypal problems in the study of complex phenomena. Although this class of flows is routinely encountered in typical applications of materials science, at present several questions remain still unanswered. This is especially true with regard to the hierarchy of bifurcations to oscillatory flow and back transitions to steady state that such systems display when the related (independent) characteristic numbers are varied in the space of parameters. The complexity of the problem is further reinforced by the sensitivity of these systems to the effective shape of the domain hosting the fluid and the related thermal boundary conditions.

Building on, but also seeking to extend, the valuable research that has highlighted the counterintuitive effect of thermal buoyancy on the typical supercritical states of Marangoni flow (thermal buoyancy leading to the unexpected mitigation and final suppression of hydrothermal 
waves), in this work, our focus has been on clarifying this issue through numerical solution of the governing equations in the their complete non-linear and time-dependent formulation.

Given the significant computational cost being associated with this approach (though partially mitigated by the possibility to conduct part of the explorative simulations in a two-dimensional framework), attention has been expressly concentrated on the analysis of potentially key aspects expected to "interfere" with the abovementioned hierarchy of spatio-temporal behaviors.

In particular, we have shown that the introduction of a blockage in the bottom of a rectangular shallow cavity wall can accelerate the process leading to flow stabilization when the Rayleigh number is increased for a fixed value of the Marangoni number. By making Ra higher, flow stabilization first occurs in the regions characterized by larger vertical depth and then it extends to the entire physical domain as this parameter grows further. For a fixed value of Ra (and Ma), a similar phenomenon can be induced by increasing the thickness of the topography.

Thermal conditions at the bottom wall do also play a role in such scenario by causing a shift of the required Rayleigh number for the back transition to steady flow to higher values when adiabatic conditions are replaced by conducting ones. Though such threshold values do not depend significantly on the horizontal location of the blockage, the position of the step has a remarkable influence on the general properties of the emerging steady flow.

Such flow is characterized by the presence of one or more segments of a "stationary wave". While the related wavenumber is essentially a function of the type of thermal boundary condition at the bottom, the effective number of appearing segments is related to the location of the topography (a primary branch spreads towards the interior from the hot wall, with other branches eventually originating in the regions where sudden changes in the cross section of the cavity occur).

As fully confirmed by the three-dimensional computations, this means that, as predicted by the absolute stability theory, travelling waves can effectively be turned into stationary disturbances originating from vertical walls or even from regions with abrupt changes in the domain shape when the relative strength of buoyancy forces exceeds a given threshold.

Future work shall be devoted to examining cavities with an obstruction on the bottom and free (top) surface allowed to exchange heat with the external environment for which possible competition between stationary transverse and longitudinal disturbances is expected (thereby potentially leading to a problem with multiple solutions, i.e. different types of flow coexisting in the space of states). The exploration could also be extended to the case of liquid metals. Moreover, some effort shall also be provided to replace the idealized (adiabatic or conducting) thermal conditions at the bottom boundary with more realistic ones accounting for the material used to manufacture the wall, its thickness and effective thermal conductivity (following an approach analogous to that undertaken by other investigators for the companion problem relating to the stability of thin films flowing down an inclined wall in the presence of thermocapillary effects [37-40]). 


\section{References}

[1] Schwabe D., Metzger J., (1989), Coupling and separation of buoyant and thermocapillary convection, J. Cryst. Growth, 97: 23-33.

[2] Saghir M. Z., Maffei N., (1999), Float zone of $\mathrm{Bi}_{12} \mathrm{GeO}_{20}$ STS 77: experimental and numerical results, Microgravity Space Station Utilization Journal, 1 (1): 31-35.

[3] Melnikov D.E., Shevtsova V.M. and Legros J.C., (2005), Route to aperiodicity followed by high Prandtl-number liquid bridge. 1-g case, Acta Astronautica, 56(6): 601-611.

[4] Melnikov D.E., Shevtsova V.M. and Legros, J.C., (2004), Onset of temporal aperiodicity in high Prandtl number liquid bridge under terrestrial conditions, Phys. Fluids, 16(5): 1746-1757.

[5] Peng L., Li Y.-R., Shi W.-Y., and Imaishi N., (2007), Three-dimensional thermocapillarybuoyancy flow of silicone oil in a differentially heated annular pool, Int. J. Heat Mass Transfer, 50(5-6): 872-880.

[6] Shevtsova V.M., Nepomnyashchy A.A. and Legros J.C., (2003), Thermocapillary-buoyancy convection in a shallow cavity heated from the side, Phys. Rev. E 67, 066308 (14 pages)

[7] Shevtsova V., Melnikov D.E., Nepomnyashchy A., (2009), New flow regimes generated by mode coupling in buoyant-thermocapillary convection, Phys. Rev. Lett, 102: 134503.

[8] Lappa M., (2009), Thermal Convection: Patterns, Evolution and Stability, John Wiley \& Sons, Ltd (2009, Chichester, England).

[9] Lappa M., (2016), On the onset of multi-wave patterns in laterally heated floating zones for slightly supercritical conditions, Phys. Fluids, 28(12): 124105 (22 pages).

[10] Gershuni G.Z., Laure P., Myznikov V.M., Roux B., Zhukhovitsky E.M., (1992), On the stability of plane-parallel advective flows in long horizontal layers, Microgravity Q., 2(3): 141-151. [11] Parmentier P.M., Regnier V.C. and Lebon G., (1993), Buoyant-thermocapillary instabilities in medium-Prandtl number fluid layers subject to a horizontal temperature gradient, Int. J. Heat Mass Transfer, 36: 2417-2427.

[12] Mercier J.F. and Normand C., (1996), Buoyant-thermocapillary instabilities of differentially heated liquid layers, Phys. Fluids, 8(6): 1433-1445.

[13] Lappa M., (2012), Exact Solutions for Thermal Problems: Buoyancy, Marangoni, Vibrational and Magnetic-Field-Controlled Flows, Review of Applied Physics, 1(1): 1-14.

[14] Smith M.K., Davis S.H. (1983), Instabilities of dynamic thermocapillary liquid layers. Part 1 : convective instabilities, J. Fluid Mech 132: 119-144.

[15] Riley R.J. and Neitzel G.P., (1998), Instability of thermocapillary-buoyancy convection in shallow layers. Part 1. Characterization of steady and oscillatory instabilities, J. Fluid Mech., 359: 143-164.

[16] Villers D. and Platten J., (1992), Coupled Buoyancy and Marangoni convection in acetone: experiments and comparison with numerical simulations, J. Fluid Mech., 234: 487-510.

[17] De Saedeleer C., Garcimartin A., Chavepeyer G., Platten J.K., Lebon G., (1996), The instability of a liquid layer heated from the side when the upper surface is open to air, Phys. Fluids, 8(3): 670-676.

[18] Ezersky A. B., Garcimartìn A., Mancini H. L., and Perez-Garcia C., (1993), Spatiotemporal structure of hydrothermal waves in Marangoni convection, Phys. Rev. E 48: 4414-4422

[19] Daviaud F. and Vince J.M., (1993), Traveling waves in a fluid layer subjected to a horizontal temperature gradient, Phys. Rev. E, 48: 4432-4436. 
[20] Gillon P. and Homsy G.M., (1996), Combined thermocapillary-buoyancy convection in a cavity: an experimental study, Phys. Fluids, 8:2953-2963.

[21] Braunsfurth M.G. and Homsy G. M., (1997), Combined thermocapillary-buoyancy convection in a cavity. Part II. An experimental study, Phys. Fluids, 9(5): 1277-1286.

[22] Garcimartìn A., Mukolobwiez N., and Daviaud F., (1997), Origin of surface waves in surface tension driven convection, Phys. Rev. E 56: 1699-1705.

[23] Pelacho M.A. and Burguete J., (1999), Temperature oscillations of hydrothermal waves in thermocapillary-buoyancy convection, Phys. Rev. E 59: 835-840.

[24] Pelacho M.A., Garcimartın A., and Burguete J., (2000), Local Marangoni number at the onset of hydrothermal waves, Phys. Rev. E, 62: 477-483.

[25] Burguete J., Mukolobwiez N., Daviaud N., Garnier N., Chiffaudel A., (2001), Buoyantthermocapillary instabilities in extended liquid layers subjected to a horizontal temperature gradient, Phys. Fluids, 13 (10): 2773-2787.

[26] Pelacho M.A., Garcimartin A., Burguete J., (2001), Travel Instabilities in Lateral Heating, Int. J. Bifurcat. Chaos, 11(11): 2881-2886.

[27] Ospennikov N. A. and Schwabe D., (2004), "Thermocapillary flow without return flow-linear flow", Exp. Fluids, 36(6 ): 938 - 945.

[28] Chan C.L. and Chen C.F., (2010), Effect of gravity on the stability of thermocapillary convection in a horizontal liquid layer, J. Fluid Mech. 647, 91-103.

[29] Sáenz P. J., Valluri P., Sefiane K., Karapetsas G., and Matar O. K., (2013), Linear and nonlinear stability of hydrothermal waves in planar liquid layers driven by thermocapillarity, Phys. Fluids 25, 094101.

[30] Sáenz P. J., Valluri P., Sefiane K., Karapetsas G., and Matar O. K., (2014), On phase change in Marangoni-driven flows and its effects on the hydrothermal-wave instabilities, Phys. Fluids 26, 024114.

[31] Kowal K., Davis S., and Voorhees P., (2018), Thermocapillary instabilities in a horizontal liquid layer under partial basal slip. J. Fluid Mech., 855, 839-859.

[32] Lappa M. and Ferialdi H., (2017), On the Oscillatory Hydrodynamic Instability of Gravitational Thermal Flows of Liquid Metals in Variable Cross-section Containers, Phys. Fluids, 29(6), 064106 (19 pages).

[33] Lappa M. and Ferialdi H., (2018), Gravitational Thermal Flows of Liquid Metals in 3D Variable Cross-section Containers: Transition from low-dimensional to high-dimensional chaos, Chaos, 28, 093114 (21 pages).

[34] Lappa M., (2018), On the Formation and Propagation of Hydrothermal waves in Solidifying Liquid Layers, Computers \& Fluids, 172, 741-760.

[35] Lappa M., (2017), Hydrothermal waves in two-dimensional liquid layers with sudden changes in the available cross-section, Int. J. Num. Meth. Heat Fluid Flow, 27 (11): 2629-2649.

[36] Lappa M., (2017), On the oscillatory hydrodynamic modes in liquid metal layers with an obstruction located on the bottom, Int. J. Thermal Science, 118: 303-319.

[37] Dávalos-Orozco L. A., (2012), The effect of the thermal conductivity and thickness of the wall on the nonlinear instability of a thin film flowing down an incline, Int. J. Nonlinear Mech. 47, $1-7$. [38] Dávalos-Orozco L. A., (2014), Nonlinear instability of a thin film flowing down a smoothly deformed thickwall of finite thermal conductivity, Interfacial Phenomena and Heat Transfer 2/1, 55 -74 . 
[39] Dávalos-Orozco L. A., (2015), Non-linear instability of a thin film flowing down a cooled wavy thick wall of finite thermal conductivity, Phys. Letters A, 379, 962 - 967.

[40] Dávalos-Orozco L. A., (2016), Thermal Marangoni instability of a thin film flowing down a thick wall deformed in the backside, Phys. Fluid 28, 054103 (12 pages).

[41] Laure P. and Roux B., (1989), Linear and non linear study of the Hadley circulation in the case of infinite cavity, J. Cryst. Growth 97(1): 226-234.

[42] Harlow F.H. and Welch J.E. (1965), Numerical calculation of time-dependent viscous incompressible flow with free surface, Phys. Fluids, 8: 2182-2189.

[43] Chorin A.J., (1968), Numerical solutions of the Navier-Stokes equations, Math. Comput., 22: 745-762.

[44] Temam R., (1968), Une méthode d'approximation de la solution des équations de NavierStokes, Bull. Soc. Math. France, 98: 115-152.

[45] Ladyzhenskaya O.A., (1969), The Mathematical Theory of Viscous Incompressible Flow, Gordon and Breach, 2nd Edition, New York - London, 1969.

[46] Choi SK, Nam HY, Cho M (1994), Systematic comparison of finite-volume calculation methods with staggered and nonstaggered grid arrangement, Numer Heat Transfer, Part B 25 (2): 205-221

[47] Choi SK, Nam HY, Cho M (1994a), Use of staggered and nonstaggered grid arrangements for incompressible flow calculations on nonorthogonal grids, Numer Heat Transfer, Part B 25(2): 193204.

[48] Lappa M., (2019), A mathematical and numerical framework for the simulation of oscillatory buoyancy and Marangoni convection in rectangular cavities with variable cross section, Chapter 12 (pp. 419-458) in Computational Modeling of Bifurcations and Instabilities in Fluid Mechanics (Springer Mathematical Series, 2018, Part of the Computational Methods in Applied Sciences book series - COMPUTMETHODS, volume 50), Editor Alexander Gelfgat - ISBN 978-3-319-91493-0. DOI: $10.1007 / 978-3-319-91494-7$.

[49] Lappa M., (2016), Patterning behavior of gravitationally modulated supercritical Marangoni flow in liquid layers, Phys. Rev. E, 93(5), 053107, (13 pages).

[50] Priede J. and Gerbeth G., (1997), Convective, absolute, and global instabilities of thermocapillary-buoyancy convection in extended layers, Phys. Rev. E, 56: 4187-4199.

[51] Pérez-García C., Madruga S., Echebarria B., Lebon G., Burguete J., (2004), Hydrothermal waves and corotating rolls in laterally heated convection in simple liquids, J. Non-Equilibrium Thermodynam., 29(4) : 377-388.

[52] Golovin A. A., Nepomnyashchy A. A., and Pismen L. M., (1997), Nonlinear evolution and secondary instabilities of Marangoni convection in a liquid-gas system with deformable interface, J. Fluid Mech., 341: 317-341.

[53] Borcia R. and Bestehorn M., (2003), Phase-field model for Marangoni convection in liquid-gas systems with a deformable interface, Phys. Rev. E, 67(6), 066307. 